\title{
PAISAJES EMERGENTES DE LAS ENERGÍAS RENOVABLES EN ESPAÑA ${ }^{1}$
}

\author{
Marina Frolova Ignatieva \\ Departamento de Análisis Geográfico Regional y Geografía Física e Instituto de Desarrollo Regional. Universidad de Granada \\ mfrolova@ugr.es \\ Cayetano Espejo Marín \\ Departamento de Geografía. Universidad de Murcia \\ cespejo@um.es \\ Eugenio Baraja Rodríguez \\ Departamento de Geografía, Universidad de Valladolid \\ baraja@fyl.uva.es \\ María José Prados Velasco \\ Departamento de Geografia Humana. Universidad de Sevilla \\ mjprados@us.es
}

\section{RESUMEN}

El objetivo de este artículo es analizar, a través del estudio de la relación entre las energías renovables y el paisaje en el contexto español, los nuevos aspectos que han de tenerse en cuenta para mejorar su ordenación. Analizamos el concepto del paisaje energético de renovables, que se ha configurado en los estudios nacionales e internacionales más recientes sobre este tema. Abordamos diferentes modalidades de los paisajes energéticos en una perspectiva comparativa. En conclusión esbozamos los principales retos para la lograr una relación más armoniosa entre las nuevas energías y el paisaje.

Palabras clave: paisaje, energías renovables, ordenación, análisis comparativo.

Fecha de recepción: octubre 2012.

Fecha de aceptación: septiembre 2014.

1 Esta publicación ha sido posible gracias a las convocatorias de Redes de Excelencia Científica del Ministerio de Ciencia e Innovación de España (Referencias CSO2009-06356-E y CSO2010-09939-E), y a los Proyectos de Investigación Fundamental No Orientada en el marco del Plan Nacional de I+D+i 2008-2011 (Referencias CSO2011-23670 y CS02011-28480). 


\section{ABSTRACT}

The objective of this article is to study the relationship between RE and the landscape in Spain so as to analyze the new aspects that must be taken into account to improve energy and landscape planning. We begin by analyzing the concept of renewable energy landscape as defined in the most recent studies on this subject, at both a national and an international level. We discuss the different forms of RE landscape from a comparative viewpoint. We then conclude by outlining the main challenges that must be faced in order to achieve a more harmonious relationship between new energies and landscape.

Key words: landscape, renewable energies, planning, comparative analysis.

\section{INTRODUCCIÓN}

Desde principios del siglo XXI se ha construido un marco muy propicio para el desarrollo de las energías renovables (EERR) en Europa, a raíz de la preocupación generalizada de responder a unos importantes retos energéticos. Estos retos refieren tanto a la sostenibilidad y a las emisiones de gases de efecto invernadero, como a la mejora de la seguridad del suministro ante las importaciones. Los gobiernos europeos han apoyado este desarrollo con una política energética que privilegió a las EERR en detrimento de las convencionales con incentivos económicos. Al mismo tiempo, algunas empresas de energía, pero también las empresas no energéticas, han participado en la implementación de esta política. En algunos casos, los incentivos han sido destinados a costear las obras de ingeniería para la construcción de plantas a cambio de las ayudas de los gobiernos; en otros, han servido para el desarrollo y fabricación de elementos para estas EERR.

No obstante las políticas europeas de las EERR de la primera década del siglo XXI han mostrado sus limitaciones. En primer lugar, no han logrado controlar el crecimiento constante de la demanda energética. En países pioneros en el desarrollo de estas energías, como es el caso de Alemania, todo lo que se ha conseguido en esta dirección ha servido únicamente para compensar el continuo crecimiento del consumo energético. En España la situación es distinta porque la demanda eléctrica ha caído en los últimos años por la crisis económica, pero hay que reconocer que las energías renovables han alcanzado un peso muy alto en potencia instalada y producción eléctrica.

En segundo lugar, las políticas de EERR tienen muchas expectativas de futuro, pero a corto plazo se ha hecho evidente que el marco político y económico de su implantación es inestable y fluctuante. Incluso los gobiernos más incentivadores han tornado a la retracción o supresión de las primas, al tiempo que vuelven a priorizar las demandas de las empresas de energía convencionales que estaban viéndose perjudicadas. Ello ha derivado en el hecho cierto de que en algunos países del sur de Europa se ha producido un crack en este sector comparable al de la burbuja inmobiliaria.

En tercer y último lugar, y a pesar de que la proliferación de instalaciones de las EERR en Europa es resultado de una política ambiental planteada a escala global -con el objetivo de luchar contra el cambio climático-, las políticas nacionales que la están aplicando son esencialmente energéticas e industriales (Le Floch y Fortin, 2011) y, salvo algunas excepciones, tienen una dimensión territorial muy débil. Generadoras de energía «limpia», las infraestructuras de las 
EERR suelen percibirse como una encarnación de la idea del desarrollo sostenible. Sin embargo su presencia, simbolizada en elementos como los aerogeneradores, ha abierto un encendido debate en muchos países en términos de su impacto paisajístico (Mercer, 2003; Frolova et al., en prensa; Le Floch y Fortin, 2011; Nadaï et al., 2011; Frolova y Pérez, 2011; Prados et al., 2012).

Los beneficios de las energías renovables son múltiples y comprobables. El aumento de generación con energías renovables por un lado, y la menor producción de las centrales térmicas, por otro, ha contribuido a reducir las emisiones de $\mathrm{CO}_{2}$ del sector eléctrico, que se han estimado para el 2013 en 61,4 millones de toneladas, un 23,1\% menos que en 2012 (Red Eléctrica de España, 2014). Se destacan igualmente otros efectos positivos, como la sostenibilidad de sus fuentes, la reducción en las emisiones contaminantes, el cambio tecnológico, la posibilidad de avanzar hacia formas de energía más distribuidas, la reducción de la dependencia energética y del déficit de la balanza comercial, o el aumento del nivel de empleo y el desarrollo rural (Ministerio de Industria, Comercio y Turismo, 2010). Se considera igualmente que son energías justas socialmente ya que suelen tener un sistema de obtención y distribución diferente de las energías tradicionales.

No obstante la transición al nuevo modelo energético está provocando unos cambios significativos en la planificación territorial de la energía y ordenación del territorio. Los costes de la transición energética son múltiples y no se miden solamente en euros y crecientes facturas por la electricidad, sino que los estudios sobre sus consecuencias también contemplan, entre otros, impactos como la reestructuración necesaria de infraestructuras energéticas; nuevas relaciones internacionales con los países que producen esta energía; nuevos usos del suelo agrícola y de las zonas marítimas, su alto impacto visual y algunos costes ambientales (Krauss et al., 2011). Numerosos estudios ponen de manifiesto que el sistema de producción de algunas de estas EERR es claramente insostenible porque continúan apoyándose parcialmente en combustibles fósiles y utilizan otros recursos como agua, suelo y paisaje. Y también porque utilizan metales raros que se extraen en China u otros países en unas condiciones de explotación de trabajadores y cuyo comercio obedece a la lógica de obtención y distribución de energías tradicionales. Al mismo tiempo, las infraestructuras ligadas a las EERR introducen nuevos aspectos en las políticas de ordenación del territorio y del paisaje, relacionados con su menor escala y carácter disperso, lo que incrementa el número de decisiones que hay que tomar sobre su emplazamiento al tiempo que aumenta la probabilidad de tensiones sobre los usos del suelo. El impacto visual relativo por megavatio (MW) de energía generada tiende a ser más alto que el de otros sistemas de energías convencionales, por su necesidad de espacio (en el caso de la energía solar y eólica) y su verticalidad (en el caso de la energía eólica). Además, las infraestructuras necesarias para su instalación, mantenimiento y canalización de la producción tienen una fuerte impronta territorial (Baraja y Herrero, 2010). A este aspecto se suma el hecho de que, en muchos casos, los mejores emplazamientos para la implantación de aerogeneradores suelen ser los lugares de mayor exposición visual. Por último, el continuo crecimiento del sector y el incremento en escala de las aplicaciones industriales de las tecnologías de energía renovable, que son cada vez más grandes y frecuentemente más caras, aumentan las probabilidades de conflictos y tensiones en diferentes países (Van der Horst y Lozada-Ellison, 2010). Las tecnologías como por ejemplo la eólica, que inicialmente tenían un tamaño de infraestructuras pequeño, tienden a incrementarlo con el objetivo de maximizar la energía disponible para vender. 
Por todo ello, a pesar de la disposición generalmente positiva hacia las energías limpias en España, las actitudes hacia proyectos concretos pueden ser completamente diferentes. Así, la resistencia hacia los generadores de las EERR por parte de algunos grupos de población española, especialmente a la eólica, han crecido en aquellas regiones donde estas instalaciones han alcanzado una alta densidad; donde existen conflictos importantes relacionados con su incompatibilidad con los usos del suelo o actividades económicas locales; o donde la población percibe estos proyectos como una forma injusta de explotación de recursos propios para el beneficio de agentes externos. Y todo ello sobre la base de argumentos de los oponentes a los proyectos que son muy variados y van más allá de las consideraciones puramente estéticas.

No cabe duda que la transición al nuevo modelo energético está teniendo un enorme impacto sobre el paisaje social, económico y geográfico, tanto en Europa en general, como en España en particular. Varios números monográficos sobre los paisajes energéticos de renovables ${ }^{2}$, libros recientes sobre las implicaciones económicas, ambientales y paisajísticas de las EERR, su planificación y aceptación social (Szarka 2007, Devine-Wright 2011, Bouneau et al 2012, Szarka et al 2012), y las sesiones especiales sobre este tema organizadas en más de una decena de recientes congresos nacionales e internacionales, atestiguan la preocupación del mundo académico por los impactos territoriales y los nuevos paisajes que emergen como expresión física de las energías renovables. A pesar de que la transición al nuevo modelo energético ha empezado en España a lo largo de la primera década de este siglo, sus efectos ya se hacen visibles en una gran parte del territorio nacional y son un reto en las nuevas prácticas de ordenación y gestión territorial. Como consecuencia de este proceso están emergiendo no solamente unos territorios y paisajes con nuevas características y nuevos actores sociales, sino también, unas nuevas relaciones entre estos actores y los territorios. Por lo tanto las EERR no solamente transforman los paisajes de España, sino también contribuyen al enriquecimiento del diálogo entre la sociedad y su territorio configurando un nuevo marco de relaciones.

El objetivo de este artículo es analizar, a través del estudio de la relación EERR/paisaje en el contexto español, los nuevos aspectos que han de tenerse en cuenta para mejorar su ordenación. Partimos de la idea que el paisaje de las energías renovables es un sistema complejo, que no se limita únicamente a su visibilidad, sino que refleja igualmente los intercambios, interrelaciones y dinámicas socioeconómicas y ambientales a diferentes escalas espacio-temporales.

En cuanto a la metodología se realiza el análisis de los instrumentos de planificación de las EERR, de los datos y documentos existentes, y se revisa bibliografía relevante española e internacional sobre los paisajes energéticos de renovables.

En primer lugar analizamos el concepto del paisaje de energías renovables que se ha configurado en los estudios más recientes sobre este tema, tanto en el contexto internacional y como en el nacional. En segundo lugar, abordamos el escenario político en el que se han implantado estas energías en España y las principales características del sector de las EERR a fecha de hoy. Por último, estudiamos las diferentes modalidades de los paisajes

2 Nimbus, 25-26, 2010, Energy Policy, 35, 2007; Landscape Research, 35 (2), 2010; Land Use Policy, 26-3, 2010; Annals of the Association of American Geographers, 101 (4), 2011. 
energéticos de renovables y sus principales problemáticas en una perspectiva comparativa, desde los más tradicionales como los de energía hidroeléctrica, hasta los más novedosos como los solares y los de bioenergías. A modo de conclusión, esbozamos los principales retos para lograr una relación más armoniosa entre las nuevas energías y el paisaje.

La investigación que ha dado lugar a este artículo ha sido posible en el marco de una serie de actuaciones y proyectos conjuntos en los que los autores vienen participando. En especial de las convocatorias de Redes de Excelencia Científicas del anterior Ministerio de Economía, Ciencia e Innovación, actualmente en suspenso. Los autores son co-fundadores de la Red Española sobre Energías Renovables y Paisaje (RESERP), que arrancó en 2010 y tuvo su continuación en 2011, y de la que se ha dado cuenta en números anteriores de esta revista (Prados, 2010a; Frolova, 2012), en los monográfico de la revista Nimbus (2010) y de Ciudady Territorio (Prados et al., 2012), y en otros trabajos en fase de edición (Frolova et al., en prensa).

\section{EL PAISAJE DE ENERGÍAS RENOVABLES: ¿UN NUEVO CONCEPTO GEOGRÁFICO?}

\section{II.1. Contexto internacional}

En el artículo de M.J. Prados et al. (2012) ya se ha realizado una revisión crítica de los trabajos internacionales sobre las EERR publicados desde finales de 1980 en adelante. Este artículo se centra en los impactos asociados a las instalaciones de energías renovables y en el análisis de los discursos sobre estas energías en el conjunto de la Unión Europea, con algunas referencias al caso de Estados Unidos. Se ha constatado que si bien los primeros trabajos se centraban solo en los impactos ambientales de las infraestructuras energéticas, a partir de la década de los noventa las aportaciones científicas sobre las energías renovables abordan también la dimensión social de su desarrollo. También son importantes los estudios enfocados al análisis de los paisajes energéticos de renovables propiamente dichos. En estos se pueden encontrar diferentes aproximaciones, siendo la más destacada la referente a las políticas de protección que se ha desarrollado desde los finales del siglo XIX. Aquí cabría destacar tres líneas de pensamiento (Bouneau y Varaschin, 2012; Frolova et al., en prensa):

- El paradigma pintoresco considera que el paisaje forma parte del patrimonio en su dimensión visual, como vedutta en pintura. Según este enfoque hay que protegerlo de interferencias visuales que pueden alterar su apariencia.

- El paradigma ambiental considera al paisaje como parte del medio ambiente, un hábitat natural para la flora y fauna. Intenta proteger este paisaje «natural» mediante gestión de unas áreas más o menos extensas (Parques Naturales y Nacionales, Reservas de Biosfera, etc.).

- El paradigma cultural considera al paisaje como resultado de interacción entre la naturaleza y la sociedad, y lo trata como una parte del medio, configurado por la sociedad con los valores y significados compartidos mediante las representaciones culturales y prácticas territoriales.

Esta tercera forma de concebir el paisaje quedó reflejada en el Convenio Europeo del Paisaje que define al paisaje como «cualquier parte del territorio tal como la percibe la población, cuyo carácter sea resultado de la acción y la interrelación de factores naturales 
y/o humanos» (Conseil de l'Europe, 2000, Art. 1). Este enfoque va más allá de la visión «experta» del paisaje, entendido como una entidad puramente material, que ha dominado durante varias décadas, incluyéndose las diferentes dimensiones de la identidad y memoria local. Esta aproximación al paisaje ha influido en la manera en la que se estudian los paisajes energéticos de renovables. Concebidos en los años 1980-1990 como espacios desnaturalizados, se perciben cada vez con más frecuencia como «contenedores» de sensibilidades, pensamientos y utopías enraizados profundamente en el territorio y como símbolos del desarrollo económico y social (Varaschin y Bouvier, 2009; Frolova et al., en prensa).

Bajo la perspectiva de sus múltiples dimensiones, los estudios recientes contemplan los paisajes energéticos de renovables como objetos heterogéneos y multifuncionales, y su análisis atiende a los distintos procesos enraizados en el ámbito local: procesos físicos, sociales, institucionales, políticos e históricos (p.ej. Varaschin y Bouvier, 2009; Nadaï y van der Horst, 2010; Frolova et al., en prensa; Bouneau y Varaschin, 2012).

\section{II.2. Los estudios sobre los paisajes energéticos de renovables en España}

En el contexto nacional, la implantación de nuevas formas de energía ilustra las tensiones y el vínculo entre energía, territorio y paisaje. El análisis de las aportaciones de la comunidad científica española nos puede dar algunas de las claves sobre el estado de la cuestión. Cabe destacar que la preeminencia anterior en las investigaciones de geógrafos españoles sobre las cuestiones energéticas en temas relacionados con la producción y el consumo de la energía en sus distintas modalidades, ha dado paso al interés generalizado hacia las interrelaciones entre los procesos energéticos y los territoriales. Las publicaciones recientes de los geógrafos españoles, como el número monográfico de la revista Nimbus dedicado a los paisajes emergentes de las energías renovables; las contribuciones al XXII Congreso de Geógrafos Españoles en la Ponencia «Energía y territorio: dinámicas y procesos» (AA.VV., 2011); los artículos de ámbito nacional publicados en algunas revistas españoles (Galdós y Madrid, 2009; Prados et al., 2012; Zografos, C. y Saladié, S., 2012) e internacionales (Torres-Sibille et al., 2009a,b; Espejo y García, 2010; Frolova, 2010; Prados, 2010b; Iglesias et al., 2011; Frolova y Pérez, 2011; de la Hoz et al., 2013); y las obras científicas y de divulgación regionales (Izquierdo, 2010; Requejo, 2010; Ardillier, 2011; Mérida y Lobón, 2012; Ghislanzoni, 2014, entre otras); reflejan la preocupación generalizada por la escala de las transformaciones territoriales y paisajísticas derivadas del desarrollo que estas fuentes de energía están experimentando en España en la última década.

En este sentido cabe subrayar la aportación de la Red Española sobre Energías Renovables y Paisaje (RESERP). En los dos seminarios celebrados en el marco de sus actividades se ha profundizado en el estudio de los paisajes energéticos de renovables y su percepción por la población para lograr una correcta inserción de las instalaciones de energías renovables en los paisajes (Prados, 2010a). Como destacan C. de Andrés y E. Iranzo (2011: 97), «la «nueva revolución energética» relacionada con el espectacular desarrollo de las energías renovables ha originado al mismo tiempo una «revolución paisajística» en ciertas zonas rurales de la Península Ibérica». Respecto a las instalaciones solares fotovoltaicas, M. Mérida y R. Lobón (2012: 11) consideran que «incorporan compleja dimensión paisajística, especialmente en aquellos territorios donde más intensa ha sido su expansión. Su localización en entornos rurales, la gran cantidad de superficie ocupada y la singularidad de sus componentes han producido importantes 
cambios paisajísticos en los lugares donde se han implantado. La transformación del paisaje se ha realizado de forma rápida y en fechas muy recientes, dejando apenas espacio para el análisis de las profundas transformaciones del paisaje que implica y para la reflexión sobre las posibles medidas de regulación y planificación territorial que se pudieran adoptar».

Si consideramos las temáticas de estas publicaciones, la mayor parte se centran en el análisis de las energías renovables en distintas modalidades (eólica, solar fotovoltáica, termosolar, biocombustibles, hidroeléctrica), aunque la energía hidroeléctrica, siempre muy presente en las aportaciones de los geógrafos españoles sobre temática energética, ha perdido algo de peso en las publicaciones. Algunos investigadores (Ibarra et al., 2011; Prados et al., 2012) indican la necesidad de cambios en las prácticas de planificación territorial de la energía renovable y de ordenación del territorio que ha de conllevar la transición al nuevo modelo energético en España. Se señala que la planificación y la gestión no van acompañadas de una valoración real de las potencialidades territoriales para la implantación de las energías renovables (Díaz et al., 2011), y que este tipo de instalaciones se han propagado en nuestro país antes de la elaboración y puesta en marcha de unos instrumentos de regulación específicos en materia de ordenación del territorio (Prados et al., 2012).

Algunos trabajos pioneros han realizado propuestas a modo de primeras tentativas de clasificación y caracterización de los paisajes energéticos de renovables (Ardiller et al., 2011; Mérida, 2012). Así, M. Mérida et al. (2009, 2010, 2011, 2012) realizan una aproximación a la caracterización de los «paisajes de renovables», centrándose en los paisajes de la energía eólica y los de la fotovoltaica en Andalucía. Estos y otros estudios sobre los impactos paisajísticos de la energía solar (Torres-Sibille et al., 2009a) y del viento (Torres-Sibille et al., 2009b) parten de su localización, emplazamiento, las características y componentes de las instalaciones y sus principales consecuencias visuales sobre el paisaje, estableciendo propuestas para su integración paisajística. Varios trabajos (Baraja y Herrero, 2010; Fernández, 2011; Herrero, 2011; Ibarra et al., 2011; Prados et al., 2012; Zografos y Saladié, 2012; Frolova et al., en prensa) exploran las formas de ocupación territorial, los conflictos y las contradicciones que acompañan el proceso del desarrollo de las energías renovables. Muchos de estos conflictos, a diferentes escalas y entre diversos agentes sociales, están relacionados con el impacto visual de los parques eólicos, especialmente cuando los aerogeneradores están ubicados en la frontera de ámbitos territoriales con «intereses» y «sensibilidades» territoriales distintas. M.P. Díaz et al. $(2010,2011)$ enfocan sus estudios en la categorización paisajística, a escala regional, a partir de las categorías establecidas en el Mapa de los Paisajes de Andalucía (2005), y proponen una zonificación del territorio andaluz para implantación de las energías renovables basada en su aptitud y potencialidad. C. de Andrés y E. Iranzo (2011) realizan un ensayo de localización, cartografía a escala nacional y tipología de algunos nuevos paisajes españoles emergentes de la energía eólica y la termosolar que llaman «Eolopaisajes» y «Heliopaisajes», combinando esta tipología con la establecida por R. Mata y C. Sanz en el Atlas de los paisajes de España (2004).

Las tendencias en la investigación española van a la par de las de otros países europeos. Es muy común que el paisaje se utilice como un argumento estético o ambiental en contra del desarrollo de los proyectos de EERR, tratándose a éstos como instalaciones industriales cuyo impacto ha de ser limitado. Mientras este enfoque tan genérico capta una parte de la realidad de las EERR, muchas veces pierde su fuerza si la cuestión se examina en profundidad. El paisaje ha sido progresivamente dotado de múltiples dimensiones y nuevos significados en 
la evaluación de proyectos energéticos (Frolova et al., en prensa). Por ejemplo, hasta ahora muchos de los trabajos españoles sobre los paisajes energéticos de renovables se han basado en los Sistemas de Información Geográfica (SIG), cuyo objetivo ha sido establecer los criterios objetivos para la localización de estas energías. Como indican D. Van der Horst y L.M. Lozada-Ellison (2010), los SIG normalmente se utilizan para examinar la visibilidad de las nuevas instalaciones en el paisaje y la extensión espacial de zonas protegidas que deben ser salvaguardadas de estos artefactos. Las capas con los datos sobre las cuencas visuales, proximidad y densidad, con los paisajes de alto valor escénico o calidad (Möller, 2010), se superponen con el fin de identificar zonas donde los parques eólicos o solares no afecten negativamente a ninguno de los elementos identificados y refuercen el sistema de abastecimiento energético (Rodríguez et al., 2011). Este enfoque se percibe por algunos investigadores como un tipo de análisis espacial multi-criterio, que solo toma en cuenta los criterios negativos (Van der Horst y Lozada-Ellison, 2010).

Otra línea de aplicación va ligada al método común de la cartografía de cuencas visuales. Las cuencas visuales son una representación limitada de la verdadera experiencia humana individual que a priori considera la visibilidad de estas infraestructuras como un elemento distorsionador (Van der Horst y Lozada-Ellison, 2010) al tiempo que relega la compleja experiencia real de relaciones sociales, económicas y culturales que la población desarrolla con la energía y su medio ambiente (Moore, 2013). Algunos estudios recientes llevados a cabo en España (Frolova et al., 2014) demuestran que los aerogeneradores no solo no se contemplan como algo problemático por la población local, sino que por el contrario, participan en la construcción de las identidades y paisajes locales. Como ejemplo de esta afirmación estarían las infraestructuras hidroeléctricas que en algunos casos se han convertido en una verdadera atracción turística y participan en la emergencia de nuevos valores y prácticas paisajísticas (Briffaud et al., en prensa).

En este contexto las metodologías cualitativas ganan cada vez más terreno en los estudios de los paisajes energéticos de renovables. Estos análisis se centran en la percepción de los paisajes de la energía renovable y su aceptabilidad (Frolova y Pérez, 2011; Frolova et al., 2014), poniendo de manifiesto la necesidad de profundizar en los estudios de percepción social de las energías renovables y mejorar los procedimientos de participación de la población local, implicando a los agentes sociales en los procesos de toma de decisiones. Como destacan Frolova et al. (2014: 77): «El descubrimiento de formas diferentes de comprensión de los paisajes locales, a través del prisma de la energía eólica, puede ser muy útil para establecer mecanismos de participación de los ciudadanos. El paisaje, como una forma compartida de concebir, experimentar y practicar las relaciones con el territorio, puede tener un rol importante en el proceso de mediación entre la población local, los promotores de proyectos eólicos y los poderes públicos, y ayudar a dar una dimensión territorial a la planificación energética, hasta ahora esencialmente industrial y cuantitativa y muchas veces desvinculada del territorio en sentido amplio».

\section{EL ESCENARIO DE LAS ENERGÍAS RENOVABLES EN ESPAÑA}

En España la incidencia de las políticas europeas de EERR ha sido muy exitosa, convirtiéndose en un país líder en la instalación de potencia eléctrica de origen renovable, sobre todo en las tecnologías de energía eólica, solar fotovoltaica y solar termoeléctrica (Tablas 1, 2 y 3). La rápida expansión de las energías renovables en España durante la primera década 
de este siglo se debe, por una parte a los desarrollos tecnológicos alcanzados en la generación de sistemas de energía renovables, y por otra a la Política de Fomento de este tipo de energías, reflejada en una serie de leyes y decretos, que han creado un marco regulatorio favorable para la implantación de las energías renovables. Los marcos regulatorios que han demostrado ser más eficaces y eficientes para el fomento de las energías renovables son aquellos que se basan en el sistema de tarifa y prima, mientras que los sistemas de incentivos fiscales o de certificados verdes negociables, solo resultan útiles como instrumento de apoyo y complemento de aquél (Peón, 2008).

Pero la implantación de las energías renovables depende no solo del contenido de la legislación y del progreso tecnológico, sino también de las condiciones en las que estas energías se están desarrollando: contexto institucional y jurídico de las decisiones y su seguimiento, grado de participación de los agentes implicados, presencia o ausencia de debates y conflictos, etc. (Frolova y Pérez, 2011). Entre estos factores culturales, contextuales, socioeconómicos, políticos y físicos complejos, se consideran como los más importantes los siguientes: el potencial geográfico; el modelo de planificación y ordenación del territorio; el sistema de soporte financiero cuya eficacia varía en función del tiempo; los valores atribuidos al paisaje y su conservación; y finalmente, el grado de participación pública y de propiedad local en los esquemas de construcción de parques eólicos (Toke et al., 2008; Wolsink, 2007). Para el contexto español, además de la diversidad y abundancia de las fuentes de energías renovables y un régimen de fuerte apoyo financiero, destacan otros factores que han favorecido la implantación de estas energías. Entre los más importantes estarían la ausencia de una oposición fuerte y eficaz a su implantación, sobre todo en el caso de la energía eólica, tan presente en otros contextos europeos, y las formas dominantes de participación pública que no implican a los agentes sociales o bien lo hacen manteniéndolos a distancia en el proceso de toma de decisiones.

Tabla 1

EVOLUCIÓN DE LA POTENCIA INSTALADA EN ESPAÑA EN ENERGÍAS RENOVABLES. RÉGIMEN ESPECIAL. 1998-2013. MEGAVATIOS

\begin{tabular}{|r|r|r|r|r|r|r|r|r|}
\hline & $\begin{array}{c}\text { SOLAR } \\
\text { FOTOVOLTAICA }\end{array}$ & $\begin{array}{c}\text { SOLAR } \\
\text { TERMOELÉCTRICA }\end{array}$ & EÓLICA & HIDRÁULICA & BIOMASA & RESIDUOS & $\begin{array}{r}\text { TRATAMIENTO } \\
\text { DE RESIDUOS }\end{array}$ & TOTAL \\
\hline 1998 & 1 & & 886 & 1.296 & 81 & 334 & 2.599 \\
\hline 1999 & 2 & 1.686 & 1.436 & 88 & 351 & 39 & 3.592 \\
\hline 2000 & 2 & & 2.296 & 1.466 & 148 & 339 & 41 & 4.331 \\
\hline 2001 & 4 & & 1.559 & 231 & 449 & 157 & 5.907 \\
\hline 2002 & 7 & & 5.066 & 1.591 & 353 & 461 & 326 & 7.806 \\
\hline 2003 & 11 & & 1.664 & 455 & 468 & 423 & 9.344 \\
\hline 2004 & 47 & & 10.095 & 1.768 & 500 & 585 & 474 & 11.791 \\
\hline 2005 & 146 & & 11.897 & 1.898 & 541 & 579 & 543 & 13.538 \\
\hline 2006 & & & 1.706 & 470 & 585 & 629 & 15.689 \\
\hline
\end{tabular}


Tabla 1 (continuación)

EVOLUCIÓN DE LA POTENCIA INSTALADA EN ESPAÑA EN ENERGÍAS RENOVABLES. RÉGIMEN ESPECIAL. 1998-2013. MEGAVATIOS

\begin{tabular}{|l|r|r|r|r|r|r|r|r|}
\hline & $\begin{array}{c}\text { SOLAR } \\
\text { FOTOVOLTAICA }\end{array}$ & $\begin{array}{c}\text { SOLAR } \\
\text { TERMOELÉCTRICA }\end{array}$ & EÓLICA & HIDRÁULICA & BIOMASA & RESIDUOS & $\begin{array}{r}\text { TRATAMIENTO } \\
\text { DE RESIDUOS }\end{array}$ & TOTAL \\
\hline 2007 & 690 & 11 & 14.536 & 1.895 & 557 & 559 & 533 & 18.781 \\
\hline 2008 & 3.398 & 61 & 16.323 & 1.979 & 587 & 569 & 571 & 23.488 \\
\hline 2009 & 3.397 & 232 & 18.861 & 2.016 & 670 & 587 & 658 & 26.421 \\
\hline 2010 & 3.837 & 532 & 19.706 & 2.030 & 709 & 627 & 658 & 28.098 \\
\hline 2011 & 4.246 & 999 & 21.069 & 2.035 & 765 & 599 & 658 & 30.370 \\
\hline 2012 & 4.538 & 1.950 & 22.636 & 2.033 & 839 & 610 & 658 & 33.263 \\
\hline 2013 & 4.640 & 2.300 & 22.785 & 2.038 & 848 & 602 & 633 & 33.846 \\
\hline
\end{tabular}

Fuente: Comisión Nacional de los Mercados y la Competencia.

Tabla 2

EVOLUCIÓN DE LA PRODUCCIÓN DE ELECTRICIDAD EN ESPAÑA CON ENERGÍAS RENOVABLES. RÉGIMEN ESPECIAL.1998-2013. GIGAVATIOS/HORA

\begin{tabular}{|c|c|c|c|c|c|c|c|c|}
\hline & $\begin{array}{c}\text { SOLAR } \\
\text { FOTOVOLTAICA }\end{array}$ & $\begin{array}{c}\text { SOLAR } \\
\text { TERMOELÉCTRICA }\end{array}$ & EÓLICA & HIDRÁULICA & BIOMASA & RESIDUOS & $\begin{array}{l}\text { TRATAMIENTO } \\
\text { DE RESIDUOS }\end{array}$ & TOTAL \\
\hline 1998 & 1 & & 1.354 & 3.622 & 180 & 1.589 & & 6.747 \\
\hline 1999 & 1 & & 2.693 & 3.794 & 200 & 1.746 & 98 & 8.533 \\
\hline 2000 & 1 & & 4.688 & 3.933 & 275 & 1.639 & 194 & 10.730 \\
\hline 2001 & 2 & & 6.930 & 4.406 & 708 & 1.960 & 549 & 14.556 \\
\hline 2002 & 5 & & 9.602 & 3.899 & 1.201 & 2.201 & 994 & 17.902 \\
\hline 2003 & 9 & & 12.060 & 5.088 & 1.585 & 2.328 & 2.070 & 23.141 \\
\hline 2004 & 18 & & 16.086 & 4.749 & 1.798 & 2.312 & 2.662 & 27.625 \\
\hline 2005 & 40 & & 21.187 & 3.817 & 2.120 & 2.620 & 3.172 & 32.955 \\
\hline 2006 & 105 & & 23.166 & 4.144 & 2.136 & 2.446 & 3.395 & 35.392 \\
\hline 2007 & 484 & 8 & 27.601 & 4.121 & 2.173 & 2.722 & 3.414 & 40.522 \\
\hline 2008 & 2.528 & 15 & 32.128 & 4.636 & 2.488 & 2.732 & 3.139 & 47.665 \\
\hline 2009 & 6.074 & 130 & 38.275 & 5.444 & 3.025 & 2.928 & 3.926 & 59.800 \\
\hline 2010 & 6.398 & 692 & 43.144 & 6.753 & 3.141 & 3.127 & 4.285 & 67.539 \\
\hline 2011 & 7.415 & 1.779 & 41.862 & 5.281 & 3.751 & 2.972 & 4.421 & 67.482 \\
\hline 2012 & 8.158 & 3.433 & 48.329 & 4.634 & 4.197 & 2.848 & 4.502 & 76.102 \\
\hline 2013 & 8.262 & 4.422 & 54.432 & 7.017 & 4.615 & 2.731 & 4.438 & 85.916 \\
\hline
\end{tabular}

Fuente: Comisión Nacional de los Mercados y la Competencia. 
Tabla 3

APORTACIÓN DE LAS ENERGÍAS RENOVABLES AL TOTAL DE LA DEMANDA DE ELECTRICIDAD EN ESPAÑA. RÉGIMEN ESPECIAL. 1998-2013. \%

\begin{tabular}{|c|c|c|c|c|c|c|c|c|}
\hline & $\begin{array}{c}\text { SOLAR } \\
\text { FOTOVOLTAICA }\end{array}$ & $\begin{array}{c}\text { SOLAR } \\
\text { TERMOELÉCTRICA }\end{array}$ & EÓLICA & HIDRÁULICA & BIOMASA & RESIDUOS & $\begin{array}{l}\text { TRATAMIENTO } \\
\text { DE RESIDUOS }\end{array}$ & TOTAL \\
\hline 1998 & $0,0 \%$ & & $0,7 \%$ & $2,0 \%$ & $0,1 \%$ & $0,9 \%$ & & $3,7 \%$ \\
\hline 1999 & $0,0 \%$ & & $1,4 \%$ & $2,0 \%$ & $0,1 \%$ & $0,9 \%$ & $0,1 \%$ & $4,4 \%$ \\
\hline 2000 & $0,0 \%$ & & $2,3 \%$ & $1,9 \%$ & $0,1 \%$ & $0,8 \%$ & $0,1 \%$ & $5,2 \%$ \\
\hline 2001 & $0,0 \%$ & & $3,2 \%$ & $2,0 \%$ & $0,3 \%$ & $0,9 \%$ & $0,3 \%$ & $6,7 \%$ \\
\hline 2002 & $0,0 \%$ & & $4,3 \%$ & $1,7 \%$ & $0,5 \%$ & $1,0 \%$ & $0,4 \%$ & $8,0 \%$ \\
\hline 2003 & $0,0 \%$ & & $5,1 \%$ & $2,1 \%$ & $0,7 \%$ & $1,0 \%$ & $0,9 \%$ & $9,7 \%$ \\
\hline 2004 & $0,0 \%$ & & $6,4 \%$ & $1,9 \%$ & $0,7 \%$ & $0,9 \%$ & $1,1 \%$ & $11,1 \%$ \\
\hline 2005 & $0,0 \%$ & & $8,1 \%$ & $1,5 \%$ & $0,8 \%$ & $1,0 \%$ & $1,2 \%$ & $12,6 \%$ \\
\hline 2006 & $0,0 \%$ & & $8,6 \%$ & $1,5 \%$ & $0,8 \%$ & $0,9 \%$ & $1,3 \%$ & $13,2 \%$ \\
\hline 2007 & $0,2 \%$ & $0,0 \%$ & $9,9 \%$ & $1,5 \%$ & $0,8 \%$ & $1,0 \%$ & $1,2 \%$ & $14,5 \%$ \\
\hline 2008 & $0,9 \%$ & $0,0 \%$ & $11,5 \%$ & $1,7 \%$ & $0,9 \%$ & $1,0 \%$ & $1,1 \%$ & $17,1 \%$ \\
\hline 2009 & $2,3 \%$ & $0,0 \%$ & $14,4 \%$ & $2,0 \%$ & $1,1 \%$ & $1,1 \%$ & $1,5 \%$ & $22,4 \%$ \\
\hline 2010 & $2,3 \%$ & $0,3 \%$ & $15,7 \%$ & $2,5 \%$ & $1,1 \%$ & $1,1 \%$ & $1,6 \%$ & $24,5 \%$ \\
\hline 2011 & $2,7 \%$ & $0,7 \%$ & $15,5 \%$ & $2,0 \%$ & $1,4 \%$ & $1,1 \%$ & $1,6 \%$ & $25,0 \%$ \\
\hline 2012 & $3,1 \%$ & $1,3 \%$ & $18,2 \%$ & $1,7 \%$ & $1,6 \%$ & $1,1 \%$ & $1,7 \%$ & $28,6 \%$ \\
\hline 2013 & $3,2 \%$ & $1,7 \%$ & $20,9 \%$ & $2,7 \%$ & $1,8 \%$ & $1,0 \%$ & $1,7 \%$ & $33,0 \%$ \\
\hline
\end{tabular}

Fuente: Comisión Nacional de los Mercados y la Competencia. http://www.cnmc.es/.

\section{III.1. Las energías renovables en España en 2013}

Al finalizar el año 2013 España cuenta con una potencia instalada para producir electricidad que asciende a 108.249 MW, el 94,5\% en centrales localizadas en el territorio peninsular (Tabla 4). Esta gran capacidad supera en más del doble el máximo de potencia instantánea que se ha producido en los últimos años. Según Red Eléctrica de España, en 2013 el máximo de potencia se registró el miércoles 27 de febrero a las 20,42 con $40.277 \mathrm{MW}$, un 7,5\% menor que el máximo registrado en 2012 de 43.527 MW, y un 11,4\% inferior al record de 45.450 MW alcanzado el 17 de diciembre de 2007. Estos datos reflejan claramente dos hechos: el impacto de la crisis económica y su incidencia sobre el consumo de electricidad en nuestro país, y por otro lado si es preciso seguir construyendo centrales.

El desarrollo de las energías renovables en España ha sido tan intensivo que llevado a que en la actualidad cerca de la mitad $(46,8 \%)$ de la potencia instalada corresponda a estas tecnologías. La eólica, implantada en la primera década de este siglo, y la gran hidráulica, desarrollada durante la segunda mitad del siglo pasado, son las principales. En el resto se incluyen las solares (fotovoltaica y termoeléctrica), minihidráulica, biomasa y residuos. 
Tabla 4

POTENCIA DE ENERGÍA ELÉCTRICA INSTALADA EN ESPAÑA. 2013. MEGAVATIOS

\begin{tabular}{|c|c|c|c|c|c|c|c|}
\hline & $\begin{array}{c}\text { Sistema } \\
\text { Peninsular }\end{array}$ & Canarias & Baleares & Ceuta & Melilla & Total MW & \% España \\
\hline Hidráulica & $17.765,0$ & 1,0 & & & & $17.766,0$ & 16,4 \\
\hline Nuclear & $7.866,0$ & & & & & $7.866,0$ & 7,3 \\
\hline Carbón & $11.131,0$ & & 510,0 & & & $11.641,0$ & 10,8 \\
\hline Fuel/gas & 520,0 & $1.918,0$ & 877,0 & 99,0 & 85,0 & $3.499,0$ & 3,2 \\
\hline Ciclo combinado & $25.353,0$ & 920,0 & 934,0 & & & $27.207,0$ & 25,1 \\
\hline $\begin{array}{l}\text { Total Régimen } \\
\text { ordinario }\end{array}$ & $62.635,0$ & $2.839,0$ & $2.321,0$ & 99,0 & 85,0 & $67.979,0$ & 62,8 \\
\hline Hidráulica & $2.102,0$ & 0,5 & & & & $2.102,5$ & 1,9 \\
\hline Eólica & $22.854,0$ & 153,0 & 4,0 & & & $23.011,0$ & 21,3 \\
\hline Solar fotovoltaica & $4.424,0$ & 165,0 & 78,0 & & 0,1 & $4.667,1$ & 4,3 \\
\hline Solar termoeléctrica & $2.300,0$ & & & & & $2.300,0$ & 2,1 \\
\hline Térmica renovable & 975,0 & 3,0 & 2,0 & & & 980,0 & 0,9 \\
\hline $\begin{array}{l}\text { Térmica no } \\
\text { renovable }\end{array}$ & $7.089,0$ & 33,0 & 86,0 & & 2,0 & $7.210,0$ & 6,7 \\
\hline $\begin{array}{l}\text { Total régimen } \\
\text { especial }\end{array}$ & $39.744,0$ & 354,5 & 170,0 & $\mathbf{0 , 0}$ & 2,1 & $40.270,6$ & 37,2 \\
\hline Total & $102.379,0$ & $3.193,5$ & $2.491,0$ & 99,0 & 87,1 & $108.249,6$ & 100,0 \\
\hline
\end{tabular}

Fuente: Red Eléctrica de España.

El elevado peso de la capacidad instalada en renovables tiene claro reflejo en su aportación a la producción total de electricidad en España (Tabla 5). Somos el país del mundo en el que la energía eólica contribuye en mayor medida a la producción total eléctrica (Espejo y García, 2012), una quinta parte en 2013. Según Red Eléctrica de España a lo largo de 2013 se han superado los niveles máximos de producción eólica alcanzados en años anteriores, El 6 de febrero a las 15,49 horas la producción eólica alcanzó los 17.056 MW (un 2,5\% superior al anterior máximo alcanzado en abril de 2012). Así mismo, en los meses de enero, febrero, marzo y noviembre la generación eólica ha sido la tecnología con mayor contribución a la producción de energía total del sistema. Cuando no hay presencia de vientos, o si incidencia es muy escasa, se recurre al amplio parque generador de las centrales de ciclo combinado, que supera en la Península los 25.000 MW, y cuyo principal problema hoy en día es su infrautilización, no solo por la prioridad de las renovables, sino por la caída de la demanda de electricidad.

En 2013 tras la eólica se sitúa la electricidad procedente de la gran hidráulica, aunque como hecho puntual, debido a su dependencia de la mayor o menor disponibilidad de agua según los años. El resto de tecnologías renovables contribuyen en menor medida porque su potencia instalada es inferior, y sobre todo porque su sistema de producción eléctrica no permite disponer de una capacidad tan grande en sus instalaciones. 
Tabla 5

PRODUCCIÓN DE ENERGÍA ELÉCTRICA EN ESPAÑA. 2013. GIGAVATIOS/HORA

\begin{tabular}{|c|c|c|c|c|c|c|c|}
\hline & $\begin{array}{l}\text { Sistema } \\
\text { Peninsular }\end{array}$ & Canarias & Baleares & Ceuta & Melilla & Total MW & \% España \\
\hline Hidráulica & 33.970 & & & & & 33.970 & 12,1 \\
\hline Nuclear & 56.827 & & & & & 56.827 & 20,2 \\
\hline Carbón & 39.807 & & 2.591 & & & 42.398 & 15,1 \\
\hline Fuel/gas & & 5.266 & 1.293 & 221 & 216 & 6.996 & 2,5 \\
\hline Ciclo combinado & 25.091 & 3.134 & 447 & & & 28.672 & 10,2 \\
\hline $\begin{array}{l}\text { Total Régimen } \\
\text { ordinario }\end{array}$ & 155.695 & 8.400 & 4.331 & 221 & 216 & 168.863 & 60,1 \\
\hline Hidráulica & 7.099 & 3 & & & & 7.102 & 2,5 \\
\hline Eólica & 54.338 & 364 & 6 & & & 54.708 & 19,5 \\
\hline Solar fotovoltaica & 7.915 & 287 & 122 & & & 8.324 & 3,0 \\
\hline $\begin{array}{l}\text { Solar } \\
\text { termoeléctrica }\end{array}$ & 4.442 & & & & & 4.442 & 1,6 \\
\hline Térmica renovable & 5.064 & 8 & 1 & & & 5.073 & 1,8 \\
\hline $\begin{array}{l}\text { Térmica no } \\
\text { renovable }\end{array}$ & 31.989 & 0 & 252 & & & 32.241 & 11,5 \\
\hline $\begin{array}{l}\text { Total régimen } \\
\text { especial }\end{array}$ & 110.847 & 662 & 381 & $\mathbf{0}$ & $\mathbf{0}$ & 111.890 & 39,9 \\
\hline Total & 266.542 & 9.062 & 4.712 & 221 & 216 & 280.753 & 100,0 \\
\hline
\end{tabular}

Fuente: Red Eléctrica de España.

\section{III.2. La evolución de las políticas de energías renovables en España}

La Ley 54/1997 del Sector Eléctrico tiene entre otros objetivos la mejora de la eficiencia energética, la reducción del consumo y la protección del medio ambiente, necesarios en función de los compromisos adquiridos por España en la reducción de gases de efecto invernadero. Esta Ley lleva a la promulgación del Real Decreto (R.D.) 2818/1998 sobre producción de energía eléctrica por instalaciones abastecidas por recursos o fuentes de energía renovables, residuos y cogeneración, que son las incluidas en el régimen especial. Se fija un sistema de incentivos temporales para aquellas instalaciones que requieren de ellos para situarse en posición de competencia en un mercado libre. Para las de energías renovables y de residuos, el incentivo establecido no tiene límite temporal. Se pueden acoger al régimen especial las instalaciones con potencia instalada inferior o igual a $50 \mathrm{MW}$.

El R.D. 436/2004 establece la metodología para la actualización y sistematización del régimen jurídico y económico de la actividad de producción de energía eléctrica en régimen especial. El R.D. 661/2007 sustituye al anterior y da una nueva regulación a este sistema de producción eléctrica. Implanta un nuevo régimen retributivo a aplicar a las instalaciones de 
energías renovables y de cogeneración, con el fin de alcanzar el 2010 los objetivos recogidos en el Plan de Energías Renovables 2005-2010, y en la Estrategia de Ahorro y Eficiencia Energética de España.

La crisis económica actual de Europa, que se manifiesta de forma más aguda en España, ha demostrado la fragilidad de las políticas europeas en la materia de energías renovables, ya que estas energías necesitan un apoyo económico y se basan en la tarifa regulada. Cuando las ayudas no funcionan bien su desarrollo se frena por completo. El caso de España es bien elocuente en este sentido. Las primas y tarifas establecidas en el R.D. 661/2007 provocaron un crecimiento espectacular en la energía solar fotovoltaica. Como se puede comprobar en la tabla 1, la potencia instalada pasa de 146 MW en 2006 a 690 MW en 2007 y 3.398 MW en 2008, lo que significa que en dos años se multiplica por 23. Con este R.D. también se inicia la instalación y desarrollo de la energía solar termoeléctrica, de la que España también ha sido pionera en desarrollo tecnológico e implantación (Espejo, 2010). Para la eólica también supone un gran apoyo y consolidación, ya que inicia su gran desarrollo a finales de la última década del siglo pasado.

La situación de crisis que vive nuestro país, y el incremento del déficit tarifario ha llevado a los Gobiernos a adoptar una serie de medidas cada vez más contundentes para/contra las energías renovables, a las que considera como las principales causantes del déficit tarifario, que es la diferencia entre la recaudación por la tarifas reguladas que fija la Administración y que pagan los consumidores por sus suministros regulados y por las tarifas de acceso que se fijan en el mercado liberalizado y los costes reales asociados a dichas tarifas. El R.D. 1578/2008 tiene como objeto el establecimiento de un nuevo régimen económico para estas instalaciones. Recorta la prima por energía producida un $29 \%$, hasta un valor más ajustado a los costes de generación y de esta manera orienta el sector de la energía solar fotovoltaica hacia la edificación frente a las instalaciones sobre suelo. Pero además introduce un aspecto que ha supuesto el principal freno para este sector: un sistema de cupos de potencia máxima a instalar cada año, 500 MW aproximadamente, hasta 2011. Durante los años sucesivos se reduce la tarifa de un modo inversamente proporcional (Garí, 2010). Para el resto de las tecnologías del régimen especial aparece en mayo de 2009 el R.D.-Ley 6/2009, con el que se introduce el cupo de potencia máxima a instalar anualmente para todas las tecnologías del régimen especial. Se crea un registro de pre-asignación de retribución en el que deben inscribirse las instalaciones de régimen especial para tener derecho a la prima establecida en el R.D. 661/2007. En el citado registro solo se podrán inscribir instalaciones hasta cubrir el objetivo de potencia para cada tipo de tecnología (según el PER vigente). Más adelante, el R.D. 1565/2010 elimina el pago de energía primada durante los 25 primeros años de la instalación, establecido en el R.D. 661/2007, y el R.D.-Ley 14/2010 modifica retroactivamente todas las tarifas reguladas e instauradas en el R.D. 661/2007 al tiempo que implanta la limitación de las horas equivalentes de funcionamiento de las instalaciones fotovoltaicas en función de la zona climática donde se ubiquen. Con el R.D. 1614/2010 se regulan y modifican determinados aspectos de carácter económico para las tecnologías eólica y solar termoeléctrica. Se establece una limitación de las horas equivalentes de funcionamiento con derecho a prima.

No acaban aquí las medidas de contención y limitación al crecimiento de las EERR en España. A comienzos de 2012 se publica el R.D. 1/2012, con el que se procede a la suspensión de los procedimientos de pre-asignación de retribución y a la suspensión de los incentivos económicos para nuevas instalaciones del régimen especial. A finales de ese año, la Ley 15/2012, 
de medidas fiscales para la sostenibilidad energética, impone un impuesto sobre el valor de la producción energética que grava la realización de actividades de producción e incorporación al sistema eléctrico español. El impuesto se exige al tipo del 7\%. En febrero de 2013 el R.D.-Ley $2 / 2013$, de medidas urgentes en el sistema eléctrico y en el sector financiero. Se indica que todas las metodologías de retribución se encuentran vinculadas al Índice de Precios al Consumo (IPC), y establece que se sustituya este índice por el IPC a precios constantes sin alimentos no elaborados ni productos energéticos, que son los que presentan más volatilidad de sus precios. También este R.D. modifica el R.D. 661/2007, ya que tras su entrada en vigor son dos las opciones de venta de la energía producida en el régimen especial: la cesión de la electricidad percibiendo una tarifa regulada o la venta de la electricidad en el mercado de producción eléctrica, sin complemento ni prima. A mediados de este año se publica el R.D. 9/2013, por el que se adoptan medidas urgentes para garantizar la estabilidad financiera del sector eléctrico. Habilita al Gobierno para aprobar un nuevo régimen jurídico y económico para la producción eléctrica en régimen especial. Éste se basará en la percepción de los ingresos derivados de la participación en el mercado, con una retribución adicional que, en caso de resultar necesario, cubra aquellos costes de inversión que una empresa eficiente y bien gestionada no recupere en el mercado.

Por último, el 27 de diciembre de 2013 se publica la Ley 24/2013 del Sector Eléctrico. Desde el Gobierno se señala que un elemento determinante para acometer esta reforma ha sido la acumulación, durante la última década, de desequilibrios anuales entre ingresos y costes del sistema eléctrico y que ha provocado la aparición de un déficit estructural. La elevada penetración de las tecnologías de producción incluidas en el denominado régimen especial de producción de energía eléctrica, ha ocasionado que su regulación singular ligada a la potencia y a su tecnología carezca de objeto. Por el contrario, hace preciso que la regulación contemple a estas instalaciones de manera análoga a la del resto de tecnologías que se integran en el mercado, y en todo caso, que sean consideradas por razón de su tecnología e implicaciones en el sistema, en lugar de por su potencia, por lo que se abandonan los conceptos diferenciados de régimen ordinario y especial. La Ley prevé la posibilidad, con carácter excepcional, de establecimiento de nuevos regímenes retributivos específicos para fomentar la producción a partir de fuentes de energía renovables, cogeneración de alta eficiencia y residuos, cuando exista una obligación de cumplimiento de objetivos energéticos derivados de Directivas europeas u otras normas del Derecho de la Unión Europea o cuando su introducción suponga una reducción del coste energético y de la dependencia energética exterior. Su artículo 26, titulado «Derechos y obligaciones de los productores de energía eléctrica», establece que la energía eléctrica procedente de instalaciones que utilicen fuentes de energía renovable y, tras ellas, la de las instalaciones de cogeneración de alta eficiencia, tendrá prioridad de despacho a igualdad de condiciones económicas en el mercado, sin perjuicio de los requisitos relativos al mantenimiento de la fiabilidad y la seguridad del sistema, en los términos que reglamentariamente se determinen por el Gobierno.

Todas estas medidas ponen en entredicho el Plan de Acción Nacional de Energías Renovables de España 2011-2020 (PANER),que prevé que para el 2020 las renovables alcancen69.844 MW (150.030 GWh): entre otras, la eólica unos 38.000 MW y la solar unos $13.445 \mathrm{MW}$ (PANER, 2010). No obstante, este período de paréntesis en el desarrollo de las renovables podría ofrecer un marco favorable para analizar las primeras lecciones del «boom» de las nuevas energías en España y sus importantes consecuencias territoriales y paisajísticas. 


\section{SINGULARIDADES DE LOS PAISAJES EMERGENTES DE DIFERENTES TIPOS DE ENER- GÍAS RENOVABLES}

La transición energética se basa en diferentes tipos de Energías Renovables. La manera en la que cada uno de ellos transforma el territorio y los valores y prácticas paisajísticas es específica. Veamos de qué manera.

\section{IV.1. Los paisajes de energías renovables tradicionales: Paisajes hidroeléctricos}

El primer sector de las renovables desarrollado en España ha sido el hidroeléctrico lo que ha permitido un intenso aprendizaje a lo largo de su desarrollo (Pillai et al., 2005; Warren et al., 2005; Frolova, 2010; Espejo y García, 2010). Al igual que en otros países de nuestro entorno, este sector energético se desarrolló en su mayor parte antes de los años ochenta, en contextos políticos, sociales y económicos muy diferentes y anteriores al actual, marcado por la emergencia de las preocupaciones sobre el cambio climático y sobre el agotamiento de petróleo («peakoil») (Warren et al., 2012).

Hasta hace dos décadas la energía hidroeléctrica se consideraba en España, como en muchos otros países, como una de las fuentes de energía más limpias. Con la creciente conciencia de los impactos ambientales de grandes centrales hidroeléctricas y embalses (Frolova, 2010), desde finales de los 90 (R.D. 2818/1998 derivado de la Ley 54/1997 del Sector Eléctrico) solo las centrales hidroeléctricas de 50 o menos MW pertenecen al Régimen Especial.

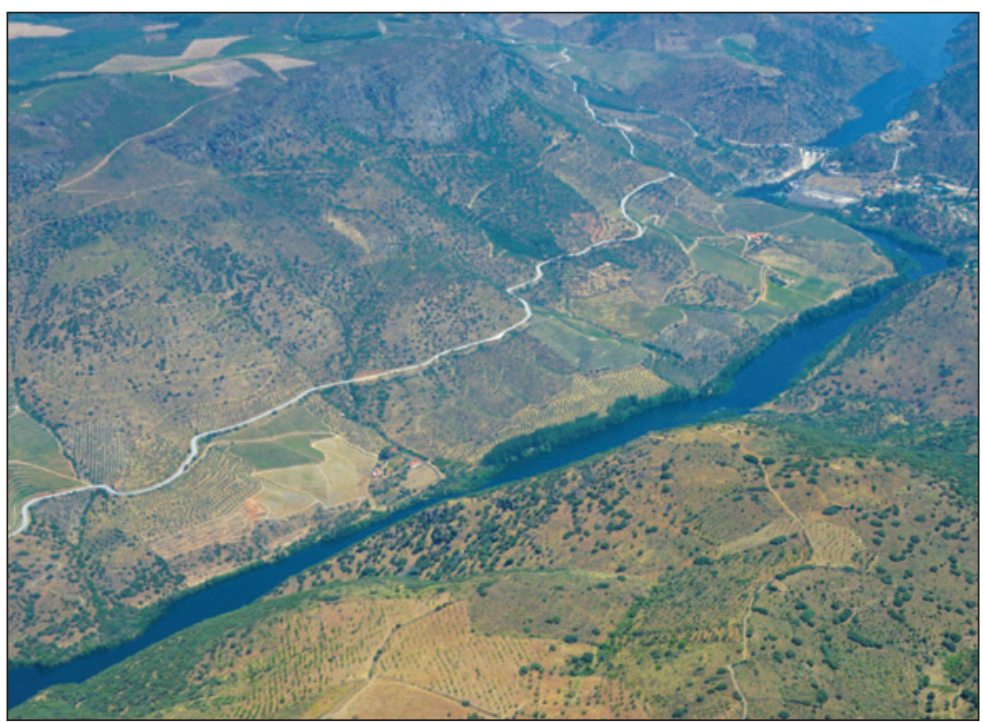

Fotografía: Eugenio Baraja. 
El contexto y las modalidades bajo las cuales se llevó a cabo el desarrollo de la energía hidroeléctrica también han sido específicos y han evolucionado a lo largo de su historia. En las primeras etapas de su desarrollo los proyectos hidroeléctricos formaron parte del proceso de electrificación, por lo que la implantación de centrales hidroeléctricas estaba íntimamente relacionada con el acceso y el consumo de la electricidad. La energía hidroeléctrica es la única tecnología que se ha desarrollado ampliamente en un contexto completamente descentralizado, cuando la producción y el consumo de energía estaban funcionando a una escala territorial local. Esto dio lugar, especialmente en zonas de montaña, al desarrollo de un conjunto de elementos específicos e interrelacionados, como centrales hidroeléctricas, embalses, presas, tuberías, canales de desviación de agua ... Estos conjuntos se perciben hoy como unos auténticos paisajes energéticos, como lo demuestran algunos casos de estudio españoles (Frolova et al., en prensa).

Estos paisajes han participado en la construcción de las identidades locales, memoria colectiva y la historia (Varaschin y Bouvier, 2009), y todo aquello contribuyó a convertir a muchos paisajes hidroeléctricas en parte del patrimonio cultural. Por lo tanto, la energía hidroeléctrica nos ofrece lecciones útiles para la comprensión de la compleja serie de relaciones, que puede subyacer la construcción de los paisajes energéticos culturalmente compartidos.

\section{IV.2. Nuevos paisajes energéticos de renovables}

El desarrollo de las nuevas tecnologías de energía como la eólica, la solar o la procedente de la biomasa, establece unas diferencias sustanciales con la hidroeléctrica.

La implantación de la energía hidroeléctrica se enmarca en una escala territorial reducida y está relacionada con la demanda de electricidad. Por el contrario, las «nuevas» energías renovables se desconectan de la escala territorial y de la demanda de energía. Se desarrollan en un momento en el que la población de los países occidentales se encuentra ya conectada a la red eléctrica. Su principal vía de implantación es a través de promotores privados (no locales), y su incorporación en el mercado, a través de primas, las hace no depender de la inversión o del consumo local. En este marco los beneficios van dirigidos a los agentes externos y los impactos van a repercutir en la población local, lo que contribuye frecuentemente a su percepción como unos proyectos ajenos al territorio que no traen unos beneficios que podrían compensar sus efectos negativos. De esta manera, las comunidades locales se sienten excluidas no solo de la construcción de estas nuevas formas de producción de energía, sino también del proceso de toma de decisiones en sí. Este carácter aparentemente impuesto de muchos proyectos de energía renovable y su percepción como un modo injusto de explotación de los recursos locales se manifiestan en diversos antagonismos, entre los «ganadores», que se aprovecharon del desarrollo de las energías renovables (empresas eléctricas, algunos ayuntamientos, propietarios de la tierra), y los «perdedores» (Frolova et al., en prensa). Estas singularidades se reflejan en la construcción de diversos paisajes de las energías renovables.

\section{IV.2.1. Los paisajes de la energía eólica}

La energía eólica forma una parte esencial de las visiones y escenarios de nuestro futuro energético, tanto a escala nacional como internacional (Baraja et al., en prensa; Warren et al., 2012). La mayor parte de escenarios futuros sobre el mix energético con- 
sidera que la energía eólica seguirá siendo el actor clave durante muchas décadas. Es la primera de las tecnologías de energía «limpia» que se han industrializado y han crecido en escala. Las ambigüedades de esta «primera tecnología renovable capitalista» también ha puesto en duda los ideales de la alternativa «blanda» (Evrard, 2012). En algunas regiones rurales, afectadas por el declive de las industrias locales y la agricultura, la reforestación continuada de las tierras agrícolas, y la disminución de la población rural, la energía eólica se asocia en ocasiones al acceso a la modernidad, al igual que la energía hidroeléctrica simbolizó la modernidad a finales del siglo XIX.

Sin embargo, la energía eólica en virtud de su desarrollo a gran escala, también ha sido la primera tecnología de energía descentralizada que «concentró riesgos en forma de enormes aerogeneradores, mientras distribuía el beneficio de la electricidad principalmente a las poblaciones lejanas que no sufrían ... de las vistas alteradas, de los cambios de uso del suelo, de impactos ambientales, del ruido, efectos ópticos, y de riesgo de accidentes asociados a estas altas estructuras» (Ottinger, 2013). Al tiempo que ha logrado materializar en el espacio rural, un nuevo orden político y económico en Europa gracias a la creciente liberalización del mercado de la electricidad y del sector eléctrico. Por lo tanto, muchos aspectos del desarrollo de la energía eólica, han hecho de esta energía un verdadero laboratorio de pruebas sobre cómo se podría descentralizar la gestión del paisaje y de la energía (Labussière y Nádai, 2014; Warren et al., 2012).

Justamente el caso de los paisajes eólicos en España corresponde a esta tendencia del desarrollo de la escala industrial, junto con el mantenimiento generalizado de los actores locales a distancia en el proceso de toma de decisiones. Su desarrollo, que cabría calificar de espectacular por la entidad e intensidad alcanzadas, está estrechamente relacionado

Figura 2

PARQUE EÓLICO EN LAS ALINEACIONES DE LA CORDILLERA CANTÁBRICA, EN EL LIIMITE ENTRE BURGOS Y CANTABRIA

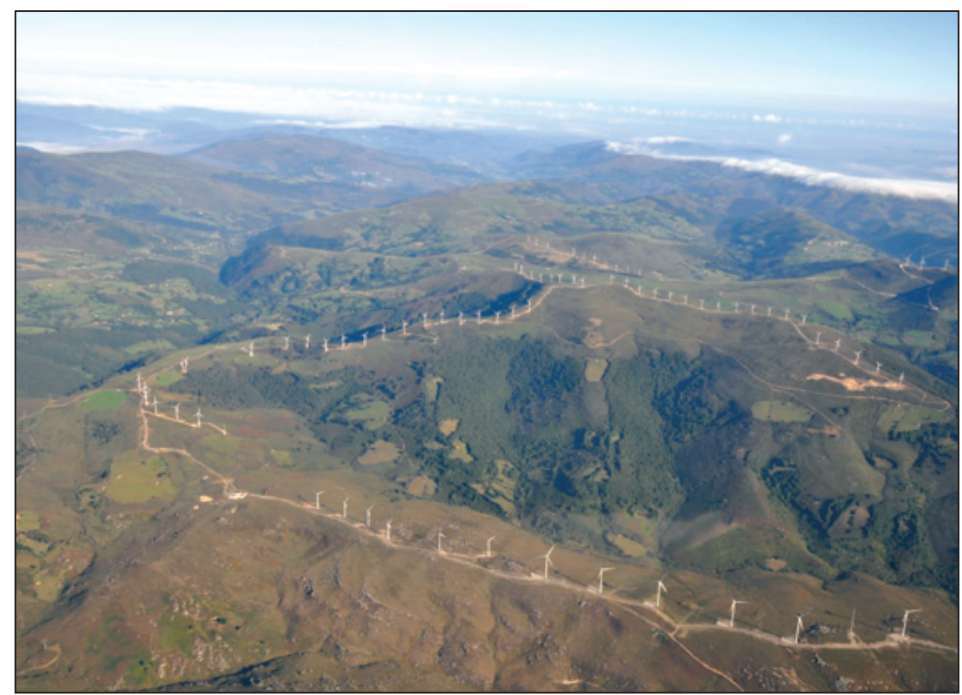

Fotografía: Eugenio Baraja. 
con la abundancia de recursos, con un marco normativo y financiero altamente estimulante -arriba descrito-, y con unos actores económicos (empresas eléctricas, industriales, inversores...) que han visto en este tipo de energía la oportunidad de un negocio de futuro. Su implantación, por otro lado, dista de ser homogénea. A las diferencias geográficas y a la diversidad paisajística del país, se le agregan los notables contrastes normativos y de «cultura territorial» en sus comunidades autónomas. Esta territorialización de la producción energética justifica que unas hayan estimulado su implantación, mientras otras han retrasado o pospuesto su desarrollo. Pero en todo caso y a diferencia de otros países europeos, la presencia de aerogeneradores ha sido, por lo común, poco contestada. De hecho, pese a su abundancia y proliferación, solo ha suscitado firme oposición y debate allí donde la densidad de aerogeneradores ha sobrepasado con creces los límites razonables; donde existen conflictos importantes relacionados con los usos del suelo (turismo, residencias secundarias...); o donde el alto valor ambiental y cultural del emplazamiento ha movilizado a los grupos sociales más sensibilizados y activos (ecologistas, científicos, plataformas ciudadanas).

Esta es una de las claves de su expansión: «un modelo donde la escasa participación ciudadana corre pareja con la actitud altamente tolerante y permisiva de la sociedad en lo que concierne a la conservación del territorio y su paisaje, lo que está muy vinculado a la consideración y valor otorgado al mismo» (Baraja et al., en prensa). Se debe aprender las lecciones del desarrollo de la energía eólica porque otros tipos de tecnologías de energías renovables, como las basadas en las fuentes marinas, solares, geotermales y biocombustibles están en la fase inicial del desarrollo a gran escala (Warren et al., 2012).

\section{IV.2.2. Paisajes energéticos solares}

Durante la primera década de este siglo la energía solar, sobre todo la fotovoltaica en suelo, también ha alcanzado la etapa de desarrollo a gran escala. Se puede afirmar el crecimiento alcanzado por el tamaño del sistema y de los artefactos; la cantidad de inversiones e incentivos; y sus impactos sobre el territorio y paisaje. El desarrollo del mercado fotovoltaico también ha llevado a la creación de un nuevo sector industrial, que solo en España en su etapa de apogeo empleaba a unas 60.000 personas (Mérida et al., en prensa). El protagonismo alcanzado en países como España ha sido tanto en el desarrollo de empresas dedicadas a la producción de energía como en la proliferación de grandes centrales de energía renovable. Y ha sido posible gracias al modelo de primas, que si bien en un principio se consideraba como un instrumento más eficaz para estimular la demanda en el desarrollo de la energía solar fotovoltaica, se mostró difícil de controlar y demasiado caro para emplear (Mentas, 2011). De ahí que el impacto de la crisis y la creciente competencia internacional han disminuido de forma significativa el crecimiento de proyectos de energía solar en toda Europa y en España en particular.

Los denominados latifundios solares de tamaño medio y a gran escala comparten algunas características con los parques eólicos: están desconectados de la escala territorial y demanda eléctrica y su impacto visual ha sido considerado como uno de los más significativos (TorresSibille et al., 2009b; de Andrés e Iranzo, 2011; Mérida, 2012). Al mismo tiempo los paisajes fotovoltaicos tienen algunas características particulares que deben ser analizadas. 


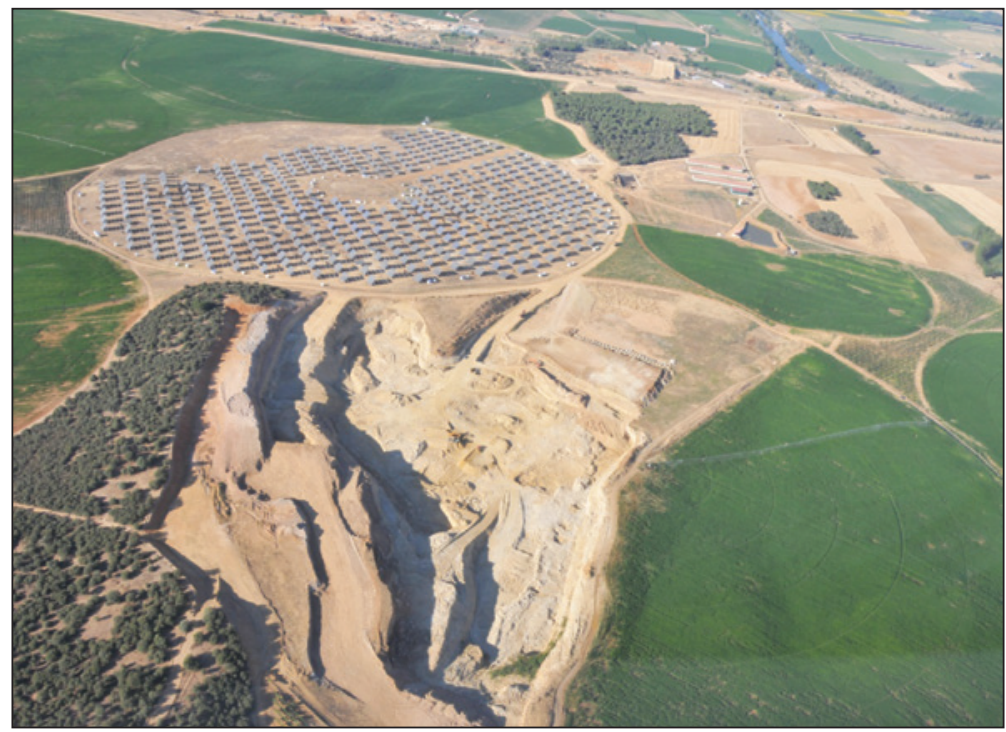

Fotografía: Eugenio Baraja.

El protagonismo de esta energía en España se debe a la disponibilidad de suelos agrícolas que han cambiado su uso a suelos para la producción energética. Esta ubicuidad en el cambio de usos de los suelos se ve favorecida porque las energías renovables tienen como características su naturaleza descentralizada y con tendencia a la dispersión (Prados, 2010). A diferencia de los parques eólicos, que son prácticamente compatibles con el uso del suelo previo a su construcción, la energía solar fotovoltaica y termoeléctrica se ha desarrollado en fincas ubicadas en áreas previamente cultivadas, por lo que representan tanto una reducción de las tierras potencialmente cultivables (Tsoutsos et al., 2005) como un cambio en el uso del suelo (Prados, 2010). El resultado ha sido la transformación de amplias franjas del territorio y el surgimiento de nuevos paisajes energéticos en las áreas rurales. Algunos investigadores consideran que la producción de electricidad en fincas agrícolas supone una competencia a la producción de alimentos, de forma similar a lo que ocurre en el caso de la producción de cultivos energéticos (Blueming et al., 2013; Chiabrando et al., 2009). El impacto de los sistemas de energía solar fotovoltaica en los cambios de uso del suelo se ve favorecido por la intensidad energética débil de la fuente solar y la baja eficiencia de la conversión de las células fotovoltaicas. Por lo tanto las dimensiones físicas de los sistemas de energía solar fotovoltaica son muy relevantes y sus impactos ambientales, territoriales y paisajísticos básicamente dependen de la extensión física del sistema (Chiabrando et al., 2009). No obstante, en investigaciones realizadas en Andalucía en relación al conocimiento ciudadano de las nuevas centrales energéticas fotovoltaicas y también de las termosolares, ha sido posible constatar que hasta la fecha éstas no provocan manifestaciones de oposición o rechazo. Actitudes que solo se hacen notar cuando se per- 
cibe una saturación de artefactos o como prevención ante posibles consecuencias nocivas para la salud o el medio ambiente (Prados 2010; de Lucas et al., 2007), lo que sin duda ha de hacerse depender de la caída de los precios agrarios, el envejecimiento de la población activa y el proceso de concentración de las explotaciones.

\section{IV.2.3. Los paisajes de los biocombustibles}

El caso especial es el de los paisajes de los biocombustibles. El biocombustible es el combustible sólido, líquido o gaseoso obtenido a partir de biomasa; y el biocarburante es el carburante líquido que se obtiene a partir de la biomasa y que, como el etanol o metanol, se puede emplear solo o mezclado con productos petrolíferos, en motores de combustión interna. La biomasa es una fuente energética renovable integrada por una variedad relativamente amplia de recursos, entre los que cabe citar los residuos agrícolas y forestales, los subproductos biodegradables, residuos industriales, etc., así como los procedentes de los cultivos agrícolas o forestales expresamente desarrollados para poder disponer de materias primas energéticas. El bioetanol es un alcohol obtenido destilando los hidratos de carbono (glúcidos, azúcares y almidones) provenientes de la materia orgánica, principalmente cereales (maíz, trigo, cebada, centeno) y cultivos con alta composición de azúcares (remolacha dulce, caña de azúcar). El bioetanol se emplea en mezclas de distinta concentración para obtener biocombustibles menos contaminantes. Con el biodiesel se procede de manera similar, pero en este caso se sustituye parte del diésel por aceites vegetales provenientes de cultivos oleaginosos, principalmente palma, soja y sorgo.

Figura 4

PLANTA DE BIOETANOL. BABILAFUENTE (SALAMANCA)

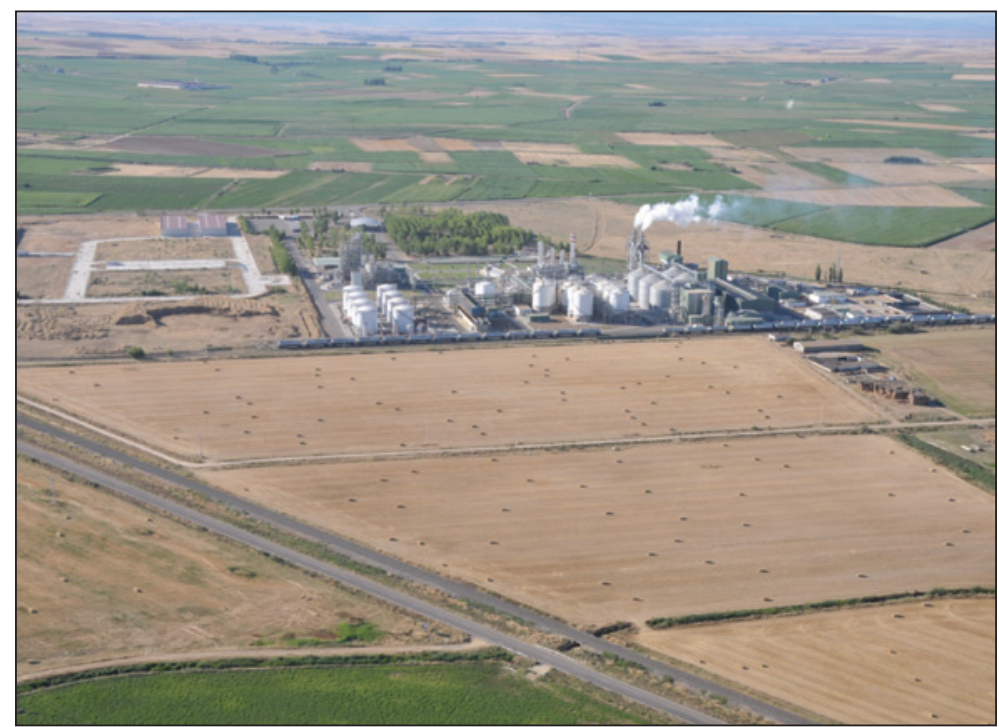

Fotografía: Eugenio Baraja. 
Figura 5

PLANTA DE BIOMASA EN LA CIUDAD DEL MEDIO AMBIENTE. GARRAY (SORIA)

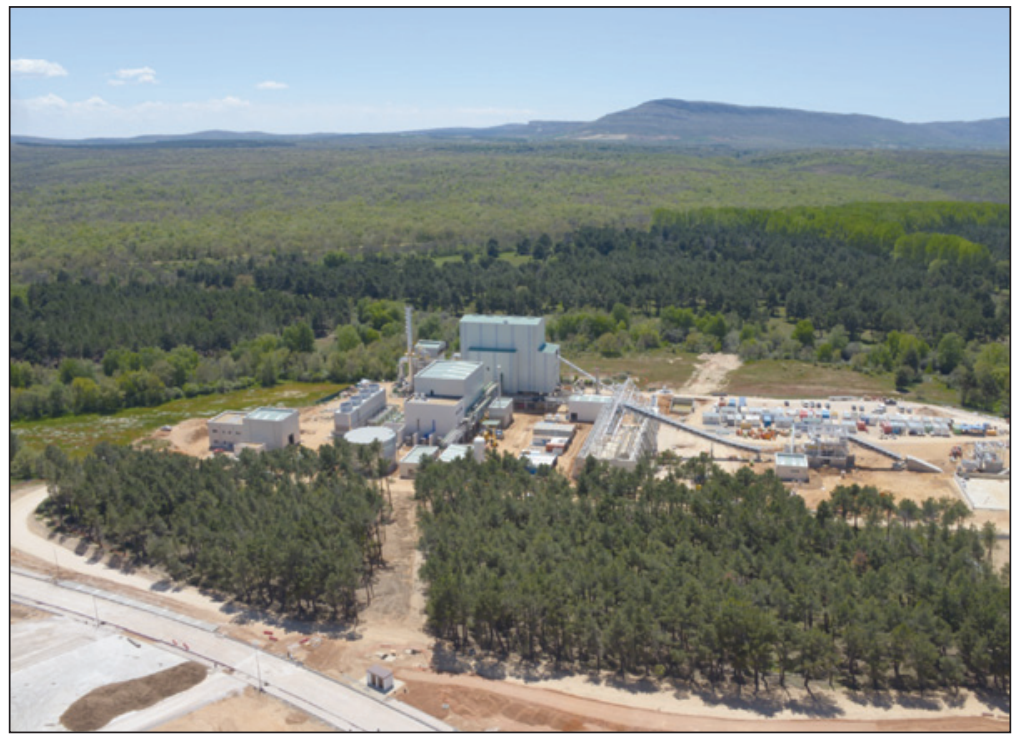

Fotografía: Eugenio Baraja.

Los países europeos están experimentando cada vez mayor producción de bioenergía. En la UE se estima que la contribución del biogás a consumo de gas natural será de $10 \%$ en 2020 (van Foreest, 2012). Solo en Italia el número de plantas de biogás ha crecido de 10 a alrededor de 1.100 entre 2012 y 2013, debido a la implantación de una política favorable con un sistema de obligaciones e incentivos (Carrosio, 2013). En España las centrales de biogás han pasado de 30 en 2001 a 34 en 2014, la potencia instalada de 449 a 602 MW, y la energía producida de 1.960 a $2.731 \mathrm{GWh}$. En este artículo solo nos centraremos en la bioenergía procedente de los cultivos agrícolas energéticos por ser el tipo de producción que más afecta a los paisajes.

Como ya se ha comentado en relación con las energías solares, las bioenergías inducen cambios de usos del suelo directos sobre los terrenos que hayan sido modificados especialmente para su producción; sin embargo, la singularidad de las bioenergías es que su implementación también induce el cambio indirecto del uso del suelo cuando la producción de biocombustibles desplaza los cultivos anteriores (Palmer, 2014), generalmente con la producción intensificada. En ambos casos la bioenergía transforma los paisajes agrícolas pre-existentes y las prácticas sociales relacionadas con ellos, aunque en el caso de cambios indirectos, esta transformación no es tan evidente como en los cambios directos de los usos del suelo. Además las bioenergías están estrechamente relacionadas no solo con la energía y las políticas ambientales (incluyendo las del paisaje), sino también con la política agrícola. A diferencia de otro tipo de fuentes de energías renovables, su producción se realiza en el marco del sector agrario y depende de sus estructuras institucionales y de las prácticas de los agricultores (Blueming et al., 2013). Y los derivados procedentes de la transformación 
de biomasa incluyen no solo energía sino también otros productos, por lo que son necesarios nuevos actores y estructuras para generar valores de estos «outputs» (Ídem.: 12). De este modo, el predominio de los productores de bioetanol a gran escala, en algunos casos, conduce a una disociación de la producción de bioetanol de la comunidad local (Bluemling et al., 2013; Carrosio, 2013). No solo la falta de coordinación entre las políticas que participan en el desarrollo de plantas de bioetanol, sino también las cuestiones más globales plantean dudas sobre sus ventajas reales tanto medioambientales y socioeconómicas (Palmer, 2014). Entre estas cabe mencionar la competencia entre energía y la producción de alimentos (por suelo y agua), la degradación del medio ambiente (a través de las emisiones de gases de efecto invernadero, la degradación del suelo y de los recursos hídricos, pérdida de biodiversidad, impacto paisajístico, etc.), y sus consecuencias sociales (a través de las infracciones en los derechos de la propiedad del suelo, o los impactos sobre la seguridad alimenticia local y regional, etc.). Además, el desequilibrio (e injusticia desde el punto de vista de la población afectada) en la distribución de los beneficios y desventajas a lo largo de la cadena de producción de bioenergías ha tenido como consecuencia la oposición de las comunidades locales a las plantas de bioetanol (Magnani, 2012; Carrosio, 2013; Ferrario y Reho, en prensa).

\section{A MODO DE CONCLUSIÓN}

En lo que va de siglo se ha construido un marco propiciatorio para el desarrollo de las energías renovables en la Unión Europea como un hito a escala global. España ha tenido una participación muy destacada en todos sus formatos, ya sea en el modo en el que ha contribuido al progreso tecnológico y a su desarrollo económico, ya sea por su amplia implantación territorial y la incidencia en nuestros paisajes. Los paisajes energéticos de las renovables se encuentran consolidados en muchos lugares, hasta el punto de que construyen identidades tanto en base a su aceptación como también en su rechazo frente a los paisajes pre-existentes. A ello ha contribuido decididamente el crack del sector energético renovable y nos sitúa en un contexto propicio para el replanteamiento de sus derivas sociales, económicas y territoriales. Con ello no pretendemos objetar los beneficios derivados de un sistema energético basado en fuentes renovables. Lo que este artículo cuestiona es que la pretendida transición al nuevo modelo energético lo haga de espaldas a unos recursos no renovables: el territorio y nuestro espacio marítimo.

El análisis de los estudios sobre las consecuencias de la implantación de los sistemas energéticos renovables en España nos demuestra que es necesario atender también a otros frentes y alcanzar retos. Algunos de los retos para lograr una relación más armoniosa entre las nuevas energías y el paisaje están todavía pendientes. Creemos que es necesario apostar por un modelo diferente de desarrollo de las energías renovables, y ello dentro de un marco que no suponga el dispendio del consumo de energía. Los desarrollos a escala de las necesidades, además de tener menores impactos paisajísticos, pueden demostrar su potencial para racionalizar producción y consumo. Y frente a las plantas fuera de escala pueden demostrar su capacidad para reducir costes; incrementar ingresos locales a largo plazo; contribuir al mantenimiento de las pequeñas explotaciones agrarias; y sin que ello suponga la reducción de la actividad económica de las áreas rurales, como sucede por ejemplo en Dinamarca y algunas regiones de Alemania (Möller, 2010; Krauss, 2010). Ello nos lleva a un segundo reto que es 
el de indagar en la relación energías renovables-desarrollo local. Se trata de un sector de creación relativamente reciente, que tiene sin embargo un peso estratégico en la economía y una enorme complejidad ya que incluye las energías de características e impactos económicos, sociales y territoriales muy diversos. De ahí que ante la ausencia de datos previos y las dificultades asociadas a su estudio se requiera el diseño de herramientas propias de análisis o bien de la adaptación cuidadosa de otras preexistentes (Arregui, 2009). Nuestra aportación futura irá en esa dirección, en la de crear una base teórica sólida para que los estudios geográficos de este sector puedan ser extrapolados y comparados. Y por último como parte nuclear de nuestra aportación, trabajaremos en el desarrollo de los instrumentos legales y criterios claros para garantizar una correcta planificación territorial y paisajística de las energías renovables.

El primer paso es reconocer que la planificación de las energías renovables está relacionada con la ausencia de verdaderas políticas territoriales y paisajísticas vinculadas a su implantación. Pese a la consolidación de la planificación territorial, al menos en el plano normativo, y a la evolución que están experimentando las políticas de paisaje, todavía hoy se encuentran desacompasadas respecto de la evolución de las políticas de energías renovables. Las prácticas y valores relacionados con el paisaje son cada vez más importantes y de cara a una mejor gestión territorial es obligado alcanzar un conocimiento más profundo de sus problemáticas. Y es que, aunque en el contexto de la UE se han llevado a cabo importantes esfuerzos en materia de paisaje y a que ello ha ido en paralelo a la necesidad de promoción de las energías renovables y a la proliferación de legislación en esta materia, no se ha conseguido introducir el paisaje de forma transversal en las políticas energéticas de los diferentes estados. La firma en 2000 y la ratificación en 2008 de la Convención Europea del Paisaje por parte de España nos insta incorporar el paisaje en la ordenación del territorio a partir del desarrollo de una legislación específica sobre paisaje de ámbito estatal que incorpore a la planificación energética de forma generalizada y transversal.

\section{REFERENCIAS BIBLIOGRÁFICAS}

AA.VV. (2011): Energía y territorio: dinámicas y procesos. Alicante, Asociación de Geógrafos Españoles, Colegio de Geógrafos de España y Universidad de Alicante.

AGULLÓ, B. y PALACÍ, J. (2011): «Energía, territorio y sociedad: zona XIV del Plan Eólico Valenciano», en Energía y territorio: dinámicas y procesos (AA.VV.). Alicante, Asociación de Geógrafos Españoles, Colegio de Geógrafos de España y Universidad de Alicante, 13-22.

ARDILLIER, F., BALABANIAN, O. y DE ANDRÉS RUIZ, C. (2011): «Los nuevos paisajes energéticos de España», en España en la Unión Europea. Un cuarto de siglo de mutaciones territoriales (Humbert, A., Molinero Hernando, F., Valenzuela Rubio, M., Ed.). Casa de Velázquez, Madrid, 41-58.

ARREGUI, G. et al. (2009): «Análisis crítico del documento «Study of the effects on employment of public aid to renewables energy sources» de G. Calzada, disponible en http://switchboard.nrdc.org/blogs/paltman/media/.

BARAJA, E. y HERRERO, D. (2010): «Energías renovables y paisaje en Castilla y León: Estudio de caso». Nimbus. Revista de Meteorología, Climatología y Paisaje, no 25-26, 21-42. 
BARAJA, E., HERRERO, D. y PÉREZ, B. (en prensa): «A Country of Windmills: Wind energy development and landscape in Spain», en Renewable Energies and European Landscapes: Lessons from Southern European cases (Frolova, M., Prados, M.J. y Nadaï, A., Ed.). New York, Springer.

BLUEMLING, B., MOL, A.P.J. y TU, Q. (2013): «The social organization of agricultural biogas production and use». Energy Policy, ${ }^{\circ}$ 63, 10-17.

BOUNEAU, Ch., VARASCHIN, D., LABORIE, L., VIGUIE, R., BOUVIER, Y. (Ed.) (2012): Les paysages de l'électricité. Perspectives historiques et enjeux contemporains (XIXe-XXIe siècles). Bruxelles, PIE Peter Lang.

BOUNEAU, Ch. y VARASCHIN, D. (2012): «Introduction» en Les paysages de l'électricité. Perspectives historiques et enjeux contemporains (XIXe-XXIe siècles) (Bouneau, Ch., Varaschin, D., Laborie, L., Viguie, R., Bouvier, Y. Ed.). Bruxelles, PIE Peter Lang, 9-20.

BRIFFAUD, S., HEAULMÉ, E., ANDRÉ-LAMAT, V., DAVASSE, B. y SACAREAU, I. (en prensa): «The nature of resources: Conflicts of landscape in the Pyrenees during the rise of hydroelectric power», en Renewable Energies and European Landscapes: Lessons from Southern European cases (Frolova, M., Prados, M.J. y Nadaï, A., Ed.). New York, Springer.

CARROSIO, G. (2013): «Energy production from biogas in the Italian countryside: Policies and organization models». Energy Policy, nº 63, 3-9.

CHIABRANDO, R., FABRIZIO, E. y GARNERO, G. (2009): «The territorial and landscape impacts of photovoltaic systems: Definition of impacts and assessment of the glare risk». Renewable and Sustainable Energy Reviews, $\mathrm{n}^{\circ} 13$ (9), 2441-2451.

CONSEIL DE L'EUROPE (2000a). Convention européenne du paysage Florence. STE, 176 (http://conventions.coe.int/Treaty/FR/treaties/html/176.htm). La traducción en español disponible en $B O E, \mathrm{n}^{\circ} 31$ (1899) de 5 de febrero de 2008, en http://otv.jccm.es/uploads/ tx_cotv/Convenio_Europeo_Paisaje.pdf.

DE ANDRÉS, C. e IRANZO, E. (2011): «Desarrollo de las energías renovables y cambios paisajísticos: propuesta de tipología y localización geográfica de los paisajes energéticos de España», en Energía y territorio: dinámicas y procesos (AA.VV.). Alicante, Asociación de Geógrafos Españoles, Colegio de Geógrafos de España y Universidad de Alicante, 97-107.

DE LA HOZ, J., MARTÍN, H., MARTINS, B., MATAS, J. y MIRET, J. (2013): «Evaluating the impact of the administrative procedure and the landscape policy on grid connected PV systems (GCPVS) on-floor in Spain in the period 2004-2008: To which extent a limiting factor?» Energy Policy, Vol. 63, 147-167.

DE LUCAS, M. (2007): Aves y parques eólicos: efectos e interacciones. Madrid, Consejo Superior de Investigaciones Científicas y Universidad Autónoma de Madrid.

DEVINE-WRIGHT, P. (Ed.) (2011). Renewable energy and the public. From NIMBY to participation. London-Washington, Earthscan Publisher.

DÍAZ, M.P., PITA, M.F. y ZOIDO, F. (2011): «El papel de la Red Eléctrica en la definición de las potencialidades territoriales para la implantación de la energía eólica en Andalucía» en Energía y territorio: dinámicas y procesos (AA.VV.). Alicante, Asociación de Geógrafos Españoles, Colegio de Geógrafos de España y Universidad de Alicante, 109118. 
DÍAZ, M.P., RODRÍGUEZ, J. y ZOIDO, F. (2010): «Energía Renovable y paisaje» en Energías renovables: Paisaje y Territorio andaluz (Izquierdo, J.M., Coord.). Sevilla, Grupo de Estudios Avanzados sobre Territorio y Medio Ambiente «Textura», 83-99.

DOMINGUEZ, J. (2011): «Los Sistemas de Información Geográfica en la frontera de la teoría y la práctica para la definición de un nuevo paisaje de las energías renovables» en Segundo Seminario de la Red Española sobre Energías Renovables y Paisaje (RESERP), Alicante, 26 de octubre de 2011, inédito.

ESPEJO, C. (2010): «Los nuevos paisajes de la energía solar: las centrales termosolares». Nimbus. Revista de Climatología, Meteorología y Paisaje, n 25-26, 65-91.

ESPEJO, C. y GARCÍA, R. (2010): «Agua y energía: Producción hidroeléctrica en España». Investigaciones Geográficas, $\mathrm{n}^{\circ}$ 51, 107-129.

ESPEJO, C. y GARCÍA, R. (2012): «La energía eólica en la producción de electricidad en España». Revista de Geografía Norte Grande, n ${ }^{\circ}$ 51, 115-136.

EVARD, A. (2013): Contre vents et marées : Politiques des énergies renouvelables en Europe. Paris, Presses de Sciences Po.

FERNÁNDEZ, J. (2011): «El efecto de las energías renovables en el paisaje vitivinícola de la Denominación de origen de Cigales», en Energía y territorio: dinámicas y procesos (AA.VV.). Alicante, Asociación de Geógrafos Españoles, Colegio de Geógrafos de España y Universidad de Alicante, 129-140.

FERRARIO, V. y REHO, M. (en prensa): «Looking beneath the landscape of carbonneutrality. Contested agroenergy landscapes in the dispersed city», en Renewable Energies and European Landscapes: Lessons from Southern European cases (Frolova, M., Prados, M.J. y Nadaï, A., Ed.). New York, Springer.

FROLOVA, M. (2010): «Landscapes, Water Policy and the Evolution of Discourses on Hydropower in Spain». Landscape Research, $\mathrm{n}^{\circ} 35$ (2), 235-257.

FROLOVA, M., BEJARANO, J. F., TORRES, A., LUCENA, M. y PÉREZ, B. (2014): «Valoración social», en Guía de integración paisajística de parques eólicos en Andalucía (Ghislanzoni, M., Ed.). Sevilla, Consejería de Medio Ambiente y Ordenación del Territorio, 65-78.

FROLOVA, M. y PÉREZ, B. (2011): «New landscape concerns in the development of renewable energy projects in South-West Spain», en Landscapes, Identities and Development (Roca, Z., Claval, P. y Agnew, J., Ed.). Farnham, Ashgate Publishing, 389-401.

FROLOVA, M., PRADOS, M.J. y NADAI, A. (Ed.) (en prensa): Renewable Energies and European Landscapes: Lessons from Southern European Cases. New York, Springer.

GALDÓS, R. y MADRID, F.J. (2009): «La energía eólica en España y su contribución al desarrollo rural». Investigaciones Geográficas, $\mathrm{n}^{\circ}$ 50, 93-108.

GARCÍA, M. (2011): «La energía como reto para la ordenación del territorio en el siglo XXI», en Energía y territorio: dinámicas y procesos (AA.VV.). Alicante, Asociación de Geógrafos Españoles, Colegio de Geógrafos de España y Universidad de Alicante, 141-152.

GARÍ, M. (Dir.) (2010): Estudio sobre el empleo asociado al impulso de las energías renovables en España 2010, Instituto Sindical de Trabajo Ambiente y Salud. Disponible en http://www.istas.net/web/abreenlace .asp?idenlace=8314, consultado 29.07.2011. 
GHISLANZONI, M. (Ed.) (2014): Guía de integración paisajística de parques eólicos en Andalucía. Sevilla, Consejería de Medio Ambiente y Ordenación del Territorio.

HERRERO, D. (2011): «La difusión de la función energética en Castilla y León: Fuerte presencia de fuentes clásicas y apuesta por las nuevas energías» en Energía y territorio: dinámicas y procesos (AA.VV.). Alicante, Asociación de Geógrafos Españoles, Colegio de Geógrafos de España y Universidad de Alicante, 153-163.

IBARRA, P., BALLARÍN, D., MORA, D., PÉREZ-CABELLO, F., ZÚÑIGA, M., ECHEVERRÍA, M.T., ALBERO, M.J. y SANTED, S. (2011): «La problemática de los parques eólicos en las áreas administrativas limítrofes: beneficio económico frente a degradación paisajística», en Energía y territorio: dinámicas y procesos (AA.VV.). Alicante, Asociación de Geógrafos Españoles, Colegio de Geógrafos de España y Universidad de Alicante, 177-189.

IGLESIAS, G., DEL RÍO, P. y DOPICO, J. A. (2011): «Policy analysis of autorization for wind energy deployment in Spain». Energy Policy, n 38, 4067-4076.

IZQUIERDO, J.M. (Coord.) (2010): Energías renovables: Paisaje y Territorio andaluz. Sevilla, Grupo de Estudios Avanzados sobre Territorio y Medio Ambiente «Textura».

KRAUSS, W., DRACKLÉ, D. y HINKELBEIN, O. (2011): «Infrastructures of German Wind Power Landscapes». International conference «Landscapes of everyday life: Intersecting perspectives on research and action», 16 - 18 Marzo de 2011, Perpignan (Francia) y Girona (España) (inédito).

LABUSSIÈRE, O. y NADAÏ, A. (2014): «Unexpected Wind Power 'Potentials': The Art of Planning with Inherited Socio-Geographical Configurations (France)». Scottish Geographical Journal. doi: 10.1080/14702541.2014.922210.

LE FLOCH, S. y FORTIN, M.J. (2011): «Le souci de l' «acceptabilité sociale» des projets énergétiques éoliens : entre consentement passif et adhésion volontaire des populations» en International conference «Landscapes of everyday life: Intersecting perspectives on research and action». 16-18 Marzo de 2011, Perpignan (Francia) y Girona (España) (inédito).

MAGNANI, N. (2012): «Exploring the local sustainability of a green economy in Alpine communities. A case study of conflict on biogas plant». Mountain Research and Development, $\mathrm{n}^{\mathrm{o}} 32$ (2), 109-116.

MERCER, D. (2003): «The great Australian wind rush and the devaluation of landscape amenity». Australian Geographer, nº 34 (1), 91-121.

MEYER, N.I. (2007): «Learning from wind energy policy in the EU: lessons from Denmark, Sweden and Spain». European Environment, n' 17 (5), 347-362.

MÉRIDA, M. y LOBÓN, R. (Coord.) (2012): Paisajes solares. Integración paisajísitca de plantas fotovoltaicas en Andalucía. Sevilla, Centro de Estudios de Paisaje y Territorio, Junta de Andalucía, CD-Rom.

MÉRIDA, M., LOBÓN, R. y PERLES, M.J. (2010): «Las plantas fotovoltaicas en el paisaje. Tipificación de impactos y directrices de integración paisajística». Nimbus: Revista de climatología, meteorología y paisaje, n 25-26, 129-154.

MÉRIDA M., LOBÓN R. y PERLES M.J. (en prensa): «The production of solar photovoltaic power and its landscape dimension in Andalusia (Spain)», en Renewable Energies and European Landscapes: Lessons from Southern European cases (Frolova, M., Prados, M.J. y Nadaï, A., Ed.). New York, Springer. 
MÉRIDA, M., LOBÓN, R., PERLES, M.J. y REYES, S. (2011): «El emplazamiento de las plantas fotovoltaicas y sus repercusiones paisajísticas», en Energía y territorio: dinámicas y procesos (AA.VV.). Alicante, Asociación de Geógrafos Españoles, Colegio de Geógrafos de España y Universidad de Alicante, 239-250.

MERIDA, M., PÉREZ, B., LOBON, R. y FROLOVA M. (2009): «Hacia la caracterización del paisaje de energías renovables» en Geografía, territorio y paisaje: el estado de la cuestión. Actas del XXI Congreso de Geógrafos Españoles (Pillet, F., Cañizares, M. y Ruiz, A., Eds.), 27-29 de octubre de 2009. Ciudad Real, Universidad de Castilla-La Mancha, 1.193-1.210.

MINISTERIO DE INDUSTRIA, COMERCIO Y TURISMO (2010): Plan de Acción Nacional de Energías Renovables en España (PANER) 2011-2020. Madrid.

MOLTÓ, E. (2011): «Informe de las características del viento en la Zona 14 y limítrofes para la instalación de aerogeneradores y acerca de los impactos paisajísticos y económicos de dicha instalación», en Energía y territorio: dinámicas y procesos (AA.VV.). Alicante, Asociación de Geógrafos Españoles, Colegio de Geógrafos de España y Universidad de Alicante, 265-276.

MÖLLER, B. (2010): «Spatial analyses of emerging and fading wind energy landscapes in Denmark». Land Use Policy, $\mathrm{n}^{\circ} 26$ (3), 233-241.

MOORE, Sh. (2013): «Envisioning the Social and Political Dynamics of Energy Transitions: Sustainable Energy for the Mediterranean Region». Science as Culture, nº 22 (2), 181188.

NADAÏ, A., KRAUSS, W., AFONSO, A. I., DRACKLÉ, D., HINKELBEIN, O., LABUSSIÈRE, O. y MENDES, C. (2010): «El paisaje y la transición energética: comparando el surgimiento de paisajes de energía eólica en Francia, Alemania y Portugal». Nimbus. Revista de Climatología, Meteorología y Paisaje, nº 25-26, 155-173.

NADAI A. y VAN DER HORST D. (2010): «Introduction». Landscape research, $\mathrm{n}^{\circ} 35$ (2), 235-257.

OTTINGER, Q. (2013): «The Wind of Change: Environmental Justice in Energy Transitions». Science as Culture, $\mathrm{n}^{\circ} 22$ (2), 222-229.

PALMER, J.R. (2014): «Biofuels and the politics of land-use change: tracing the interactions of discourse and place in European policy making». Environment and planning $A, \mathrm{n}^{\circ} 46$, 337-352.

PEÓN TORRE, J. (2008): «Desarrollo de fuentes renovables», en Energía y regulación en Iberoamérica (García Delgado, J.L. y Jiménez, J.C., Dir.). Cizur Menor, ThomsonCivitas y Comisión Nacional de Energía, 483-499.

PILLAI, A., REID, C.T. y BLACK, A.R. (2005): «Reconciling renewable energy and the local impacts of hydro-electric development». Environmental Law Review, ${ }^{\circ}$ 7, 110-123.

PRADOS, M.J. (2010a): «Red Española de Energías Renovables y Paisaje (RESERP)». Boletín de la Asociación de Geógrafos Españoles, $\mathrm{n}^{\circ}$ 52, 423-430.

PRADOS, M.J. (2010b): «Renewable energy policy and landscape management in Andalusia, Spain: the facts». Energy Policy, n 38 (11), 6.900-6.909.

PRADOS, M.J., BARAJA, E., FROLOVA, M. y ESPEJO, C. (2012): «Integración paisajística y territorial de las energías renovables». Ciudad y Territorio, vol. XLIV, $\mathrm{n}^{\circ} 171$, 131-147. 
RED ELÉCTRICA DE ESPAÑA (2014): El sistema eléctrico español 2013. Madrid. REQUEJO, J. (Coord.) (2010): Andalucía Renovable. Sevilla, Junta de Andalucía.

RODRIGUEZ, M., DOMÍNGUEZ, J., PRADOS, M. J. y VÁZQUEZ A. (2011): «Estudio del potencial energético renovable de la Isla de Cuba», en Energía y territorio: dinámicas y procesos (AA.VV.). Alicante, Asociación de Geógrafos Españoles, Colegio de Geógrafos de España y Universidad de Alicante, 399-410.

SUAREZ, J.L. y RODRÍGUEZ, J.C. (2010): «Política marítima, planificación eólica marina y energía offshore. El caso de Alborán y Golfo de Cádiz», en Energías Renovables: Paisaje y Territorio andaluz. Sevilla, Grupo de Estudios Avanzados sobre Territorio y Medio Ambiente «Textura», 119-138.

SZARKA, J. (2007): Wind Power in Europe: politics, business and society. Basingstoke, Palgrave MacMillan.

SZARKA, J., COWELL, R., ELLIS, G., STRACHAN, P. y WARREN, CH. (Ed.) (2012): Learning from Wind Power: Governance, Societal and Policy Perspectives on Sustainable Energy. Palgrave, MacMillan.

TOKE, D., BREUKERS, S., WOLSINK, M. (2008): «Wind power deployment outcomes: how can we account for the difference?». Renewable and Sustainable Energy Reviews, $\mathrm{n}^{\mathrm{o}} 12,1.129-1.147$.

TSOUTSOS, T., FRANTZESKAKI, N., GEKAS, V. (2005): «Environmental impacts from the solar energy technologies». Energy Policy, 33, 289-296.

TORRES-SIBILLE, A. C., CLOQUELL-BALLESTER, V.A., CLOQUELL-BALLESTER, V.A. y ARTACHO RAMÍREZ, M. A. (2009a): «Aesthetic impact assessment of solar power plants: An objective and a subjective approach». Renewable and Sustainable Energy Reviews, ${ }^{\circ}$ 13, 986-999.

TORRES-SIBILLE, A. C., CLOQUELL-BALLESTER, V.A., CLOQUELL-BALLESTER, V.A. y DARTON, R. (2009b): «Development and validation of a multicriteria indicator for the assessment of objective aesthetic impact ofwind farms». Renewable and Sustainable Energy Reviews, ${ }^{\circ} 13,40-66$.

VAN DER HOST, D. y LOZADA-ELLISON, L.M. (2010): «Conflictos entre las energías renovables y el paisaje: siete mitos y la propuesta de manejo adaptativo y colaborativo». Nimbus. Revista de Climatología, Meteorología y Paisaje, n 25-26, 231-251.

VARASCHIN D. y BOUVIER Y. (2009): «L'électricité, diva à Divonne» en D. Varaschin et Y. Bouvier (Ed.): Le patrimoine industriel de l'électricité et de l'hydroélectricité, Actes du colloque international de Divonne-les-Bains et de Genève, Chambéry, Université de Savoie, 7-12.

WARREN, C.R., COWELL, R., ELLIS, G., STRACHAN, P.A. y SZARKA, J. (2012): «Wind power: Towards a Sustainable Energy Future?» en Learning from Wind Power: Governance, Societal and Policy Perspectives on Sustainable Energy (SZARKA, J. et al., Ed.). Palgrave, MacMillan, Basingstoke, 1-14.

VAN FOREEST, F. (2012): «Perspectives for biogas in Europe. University of Oxford. Oxford Institute for Energy Studies». Working Paper NG70. Disponible en http://www.oxfordenergy.org/wpcms/wp-content/uploads/2012/12/NG-70.pdf.

WARREN, C.R., LUMSDEN, C., O'DOWD, S. y BIRNIE, R.V. (2005): «Green on green»: public perceptions of wind powers in Scotland and Ireland». Jornal of Environmental Planning and Management, $\mathrm{n}^{\circ} 48$ (6), 851-873. 
WOLSINK, M. (2007): «Planning of renewables schemes: Deliberative and fair decisionmaking on landscape issues instead of reproachful accusations of non-cooperation». Energy Policy, $\mathrm{n}^{\circ}$ 35, 2.692-2.704.

ZOGRAFOS, C. y SALADIÉ, S. (2012): «La ecología política de conflictos sobre energía eólica. Un estudio de caso en Cataluña». Documents d'Anàlisi Geogràfica, n ${ }^{\circ}$ 58-1, 177 192. 